\title{
Zivilstatistik: Konzept einer neuen Perspektive auf Data Literacy und Statistical Literacy
}

\author{
Joachim Engel · Rolf Biehler • Daniel Frischemeier $\cdot$ Susanne Podworny • \\ Achim Schiller $\cdot$ Laura Martignon
}

Eingegangen: 2. Juli 2019 / Angenommen: 5. November 2019 / Online publiziert: 8. November 2019 (C) Der/die Autor(en) 2019, korrigierte Publikation 2019

Zusammenfassung Demokratie lebt von Argumenten, die auf nachweisbaren Fakten basieren. Lebendige Demokratien brauchen gut informierte Bürger, die relevante gesellschaftliche Fragen verstehen, diskutieren und sich in öffentliche Entscheidungsprozesse einbringen können. Dieser Aufsatz stellt in Erweiterung von Statistical Literacy eine Teildisziplin vor, die wir Zivilstatistik nennen. Zivilstatistik konzentriert sich auf das Verstehen statistischer Informationen über die Gesellschaft, wie sie von den Medien, Statistikämtern und anderen Statistikanbietern bereitgestellt werden. Die Herausforderung, Menschen zu befähigen, Sinn aus diesen Daten zu erschließen, richtet sich gleichermaßen an Bildungseinrichtungen (Schulen und Hochschulen) wie auch an Medien und Statistikanbieter. Im vorliegenden Aufsatz beschreiben wir die spezifischen Charakteristika von Zivilstatistik und begründen die Notwendigkeit dieser Teildisziplin, die im Schnittfeld von Statistik, Gesellschaftswissenschaften und - wegen ihres Bildungsauftrages - Erziehungswissenschaften

J. Engel $(\bowtie) \cdot$ A. Schiller $\cdot$ L. Martignon

Pädagogische Hochschule Ludwigsburg, Ludwigsburg, Deutschland

E-Mail: engel@ph-ludwigsburg.de
A. Schiller
E-Mail: schiller01@ph-ludwigsburg.de
L. Martignon
E-Mail: martignon@ph-ludwigsburg.de
R. Biehler $\cdot$ D. Frischemeier $\cdot$ S. Podworny
Universität Paderborn, Paderborn, Deutschland
R. Biehler
E-Mail: biehler@math.upb.de
D. Frischemeier
E-Mail:dafr@math.upb.de
S. Podworny
E-Mail: podworny@math.upb.de 
liegt. Wir beschreiben ein Rahmenkonzept für Kompetenzen im Bereich Zivilstatistik und weisen auf reichhaltiges, frei verfügbares Lehr- und Lernmaterial hin, das im Rahmen einer europäischen Zusammenarbeit von sechs Hochschulen im Projekt ProCivicStat erarbeitet wurde. Aus unseren Analysen ergeben sich curriculare und bildungspolitische Empfehlungen, die diesen Aufsatz abschließen.

Schlüsselwörter Statistisches Denken · Multivariates Denken · Aktive Staatsbürgerschaft · Offene Daten · Curriculum

JEL-Klassifikation A21 $\cdot$ A22 $\cdot$ C00 $\cdot$ I21

\title{
Civic Statistics: Concept of new perpectives on data literacy and statistical literacy
}

\begin{abstract}
Democracy thrives on arguments based on evidence. Misinformation, absence of information and ignorance are all threats to our way of life. Vivid democracies need well-informed citizens who can understand important social issues, discuss them and contribute to public decision-making. This article introduces in extension of statistical literacy a sub-discipline we call Civic Statistics. Civic Statistics focuses on understanding statistical information about society, as provided by the media, statistical offices and other statistical providers. The challenge of empowering people to make sense of these data is addressed equally to educational institutions (schools and universities) as well as to media and statistics providers. In this article we describe the specific characteristics of Civic Statistics and explain the necessity of this subdiscipline, which is located in the intersection of statistics, social sciences and-because of its educational mission-educational sciences. We describe a framework for Civil Statistics competences and highlight rich, freely available teaching and learning material developed as part of an European collaboration between six universities in the ProCivicStat project. Our analyses provide curricular and educational recommendations that conclude this article.
\end{abstract}

Keywords Statistical thinking $\cdot$ Multivariate thinking $\cdot$ Active citizenship $\cdot$ Open data $\cdot$ Curriculum

Software. Common Online Data Analysis Platform: https://codap.concord.org/

Fathom,deutsche Version (Freeware): www.stochastik-interaktiv.de

Gapminder: https://www.gapminder.org

INZIGHT: https://www.stat.auckland.ac.nz/ wild/iNZight/index.php

Tinkerplots: https://www.tinkerplots.com

Faktenprüfende Organisationen. PolitiFact (USA) http://www.politifact.com/ Full Fact (Vereinigtes Königsreich) https://fullfact.org https://fullfact.org/blog/2017/apr/factchecking-toolkit-facebook-top-tips/ Faktenfinder (Deutschland, ARD) https://www.tagesschau.de/faktenfinder/ Für Afrika Africa Check (Afrika). https://africacheck.org/ Chequeado (Südamerika). 


\section{Einführung}

Wird die Kluft zwischen Arm und Reich größer? Sind Frauen in ihrer Karriere benachteiligt? Wie verbreitet sind rassistisches Verhalten und Fremdenfeindlichkeit in unseren Gesellschaften? Lebendige Demokratien brauchen gut informierte Bürger, die wichtige gesellschaftliche Fragen verstehen und diskutieren können. In einer zunehmend komplexeren Welt ist das Engagement von Bürgern eine grundlegende Ressource bei öffentlichen Entscheidungen auf internationalem, nationalem wie lokalem Niveau. In Anlehnung an den Begriff „Statistical Literacy“ hat die vom EU-Erasmus+ Programm geförderte internationale Kooperation ProCivicStat (unter Leitung des erstgenannten Autors und Beteiligung der weiteren Autoren und der Universitäten Durham, Haifa, Paderborn, Porto und Szeged sowie der Pädagogischen Hochschule Ludwigsburg, http://iase-web.org/islp/pcs/) ein als Zivilstatistik bezeichnetes erweitertes Konzept von Statistical Literacy entwickelt, in dessen Mittelpunkt die Sinnerschließung aus Daten steht, die über gesellschaftliche Vorgänge, das soziale und ökonomische Wohlergehen sowie die Wahrnehmung von Bürgerrechten informiert. Die Autoren dieses Aufsatzes stellen das deutsche PCS-Team dar. Das Verständnis solcher Themen ist für das bürgerschaftliche Engagement in modernen Gesellschaften von großer Bedeutung, basiert aber oft auf komplexen multivariaten Daten, deren Interpretation und Erschließung Kenntnisse voraussetzt, die in der gewöhnlichen Grundbildung in Mathematik und Statistik nicht vermittelt werden - weder an Schulen noch an Hochschulen -, geschweige denn in schulischen Fächern wie Politik oder Gemeinschaftskunde. Statistik ist zwar Teil universitärer Studienfächer wie Wirtschafts- und Sozialwissenschaften, aber nicht unter der Perspektive einer Allgemeinbildung oder eines bürgerschaftlichen Engagements. Auf der universitären Ebene gibt es in jüngster Zeit ähnliche Initiativen in einem allgemeinbildenden Sinne Komponenten der Data Literacy einzuführen (vgl. die Initiativen des Stifterverbandes https://www.stifterverband.org/data-literacy-education, Ridsdale et al. 2015; Heidrich et al. 2018).

Kompetenzen im Bereich Zivilstatistik sind zur informierten Partizipation in demokratischen Gesellschaften nötig. Bürger müssen in der Lage sein, statistische Informationen in Form von Tabellen, Graphiken und Statistiken zu zentralen gesellschaftlichen Phänomenen kritisch zu lesen und zu verstehen, um fundierte Entscheidungen im privaten wie öffentlichen Leben treffen zu können. Überparteiliche Organisationen stellen heutzutage eine Fülle von Daten frei zur Verfügung, die jeder für Informationen und Debatten nutzen kann - von den nachhaltigen Entwicklungszielen der Vereinten Nationen, der Messung des sozialen Fortschritts durch staatliche Statistikämter, die Informationen über Beschäftigung, Einkommen, Migration und vieles weitere erheben, bis hin zu Nicht-Regierungsorganisationen, die Verletzungen von Menschenrechten oder den Klimawandel und ökologische Belastungen für die Bürger dokumentieren. Technologie ermöglicht, leistungsfähige Werkzeuge zur Beschaffung und Visualisierung komplexer Daten zu nutzen, um eigenständig reichhaltige Informationsquellen zu erkunden.

Woran es mangelt, ist die Fähigkeit, solche Daten zu verstehen und aus ihnen Sinn zu erschließen. Der großen Informationsfülle stehen allerdings auch Tendenzen entgegen, die das Konzept einer evidenzbasierten politischen Entscheidungsfindung in 
Frage stellen. In Zeiten von „Fake News“ wird die Wichtigkeit von Evidenzorientierung zusehends zugunsten politischer Rhetorik von bestimmten Kreisen der politische Verantwortlichen bestritten. Es gibt eine steigende Flut von Desinformationen, oft aus politischen Gründen. Verantwortliche Journalisten werden als Volksfeinde dargestellt, Regierungsbehörden dürfen keine Rohdaten melden, die dem politischen Dogma widersprechen könnten (z. B. zum Klimawandel) ${ }^{1}$. Die leichte Verfügbarkeit von Deep-fake-Videos stellt eine weitere Bedrohung dar ${ }^{2}$. Fehlinformationen, Fake News, Informationsmangel und Ignoranz sind Bedrohungen für unsere Lebensweise. Bürger müssen kritische Fähigkeiten bei der Bewertung von vorgebrachter Evidenz entwickeln. Das bedeutet aber nicht einfach ,blind“ zu kritisieren. Es besteht die Gefahr, dass eine kritische Haltung in Zynismus über alle Datenquellen entartet, wie es etwa in den viel zitierten Worten „Lügen, verdammte Lügen und Statistiken“ zum Ausdruck kommt. Bei Kritik geht es vielmehr darum, die Haltung eines fairen Skeptikers einzunehmen, der bereit ist, einen Bericht anzunehmen, der jedoch durch Belege überzeugt werden muss (Ridgway et al. 2017). Die Idee, dass Bildung kritisches Denken fördern sollte, ist weder neu (z. B. Freire 1973), noch eine revolutionäre Idee in der Statistikausbildung. Zum Beispiel beschreibt Watson (2006) eine Hierarchie der Stufen statistischer Kompetenz, bei der auf höchster Niveaustufe Schülerinnen und Schüler ein kritisch hinterfragendes Engagement zeigen müssen, das Techniken, sprachliche Feinheiten und Argumente aus dem jeweiligen Kontext umfasst. Zivilstatistik basiert auf kritischer Bewertung und Reflexion, insbesondere auch bezüglich der Quellen und der Glaubwürdigkeit und Methoden der Datenerhebung, und sie ist ein wesentliches Mittel, um falsche Behauptungen und gefälschte Nachrichten aufzudecken.

Wie können wir junge Menschen als Staatsbürger darauf vorbereiten, statistische Daten und Erkenntnisse über Trends und Veränderungen zu wichtigen gesellschaftlichen Fragen wie demographischer Wandel, Klimaveränderung, Arbeitslosigkeit, Lohngleichheit, Migration, Gesundheit, Rassismus und andere für die Gesellschaft wichtige Bereiche zu verstehen? Zivilstatistische Daten haben spezifische Merkmale und ihr Verstehen verlangt Kompetenzen, auf die unsere Bildungseinrichtungen und Curricula nur ungenügend vorbereiten.

In unseren modernen Massengesellschaften sind Wissen und Fähigkeiten, um angemessen mit Daten zu argumentieren, eine wichtige Voraussetzung für das Funktionieren der Demokratie. In einer Gesellschaft, die den Anspruch von Gleichheit und Gerechtigkeit gegenüber all ihren Bürgern hat, können Fragen, ob z. B. Frauen, Minderheiten oder Menschen mit Behinderungen in ihren Karriereoptionen oder dem Zugang zu Bildung benachteiligt sind, weitgehend nur auf der Basis quantitativer Erhebungen beurteilt werden, weil jeder Einzelfall mit Besonderheiten behaftet sein mag. In einer zunehmend komplexen Welt ist der Beitrag der Bürger eine wichtige Ressource für politische Entscheidungsträger auf nationaler und lokaler Ebene. Die Umsetzung schwieriger Entscheidungen zu umstrittenen sozialen Themen hängt von der Zustimmung und Unterstützung der Bürger ab. Wenn die Bürger die Ent-

\footnotetext{
1 https://www.spiegel.de/wissenschaft/natur/donald-trump-umweltbehoerde-epa-stellt-klimawandelsite-voruebergehend-offline-a-1145508.html.

2 https://www.arte.tv/de/videos/091544-000-A/deepfake-die-neue-form-der-manipulation/.
} 
scheidung nicht selbst verstehen, geht das Vertrauen in politische Entscheidungen leicht verloren (OECD 2009). Eine fundierte, faktengestützte Entscheidungsfindung im privaten wie im öffentlichen Leben erfordert daher quantitative Denkfähigkeiten und - mindestens ebenso wichtig - positive Einstellungen, d.h. die Bereitschaft, sich mit statistischen Daten auseinanderzusetzen.

Dieser Beitrag will einige Charakteristika und Kompetenzanforderungen im Bereich von Zivilstatistik einführen, typische Beispiele präsentieren und auf curriculare Implikationen hinweisen. Er will ferner einem Einstieg in die Thematik dienen, Überblickswissen bereitstellen und auf Zusammenhänge hinweisen. Tiefer gehende Details zu diesem Thema sind weiteren Publikationen vorbehalten. Für den interessierten Leser sind umfangreiche weitere Ressourcen bestehend aus Lehrmaterialien, Datensätzen, konzeptionellen Darstellungen und vieles andere mehr frei in mehreren Sprachen verfügbar über die Website http://iase-web.org/islp/pcs.

\section{Was will Zivilstatistik?}

\subsection{Herausforderungen für die Bildung}

In manchen Teilen der Welt werden Statistiken über den Zustand der Welt als oberstes Staatsgeheimnis gehütet. Aber Demokratie funktioniert nur, wenn die Bürger gut informiert sind und auf der Basis von Fakten argumentieren können. Bereits 1792 machte Condorcet (1994) geltend, wie wichtig es sei, die Bürger über das Regieren zu informieren und Fakten über den Zustand der Gesellschaft vorzulegen, um das Bewusstsein für Ungerechtigkeiten und strukturelle soziale Ungleichheiten zu schärfen. Er glaubte an savoir liberateur - Wissen, das es Menschen ermöglicht, sich von sozialer Unterdrückung zu befreien. Dieses Grundprinzip ist angesichts der Datenflut und neuer Technologien zu ihrer Verarbeitung in der aktuellen Situation neu $\mathrm{zu}$ interpretieren.

Im Mittelpunkt von Zivilstatistik stehen die Verwendung von Evidenz bei der Politikgestaltung und die Notwendigkeit, dass informierte Bürger komplexe Nachweise von Evidenz verstehen und somit zu politischen Debatten beitragen können. Zivilstatistik ist Statistik zu wichtigen gesellschaftlichen Trends und Themen, die für das soziale und wirtschaftliche Wohlergehen der Bürger von Bedeutung sind. Es befindet sich an der Schnittstelle mehrerer Disziplinen, darunter Sozialwissenschaften, Erziehungswissenschaft (inklusive der Fachdidaktik) und Statistik. Daher ist eine multidisziplinäre Bildungsperspektive erforderlich, die die Komfortzone eines traditionellen, auf methodische Verfahren fokussierten Statistikunterrichts verlässt. Evidenz-informierte Entscheidungsfindung ist nicht geradlinig. Die Behandlung sozialer Themen wie Armut, Ungleichheit, Rassismus und Migration im Unterricht stellt Lehrerinnen und Lehrer vor eine Reihe von Herausforderungen. Der Zugang zu Daten kann problematisch sein, anspruchsvolle statistische Themen wie Nichtlinearität, Interaktion und multivariate Analysen müssen behandelt werden, und detailliertes Hintergrundwissen aus dem Sachkontext ist erforderlich. Oft gibt es keine „richtigen“ Antworten und gerade dieser Sachverhalt motiviert die Behandlung dieser Fragen unter dem Mantel einer neuen „Disziplin“, jenseits der Sozial- und 
Abb. 1 Zivilstatistik im Schnittfeld von Statistik, Gesellschaftsund Erziehungswissenschaft

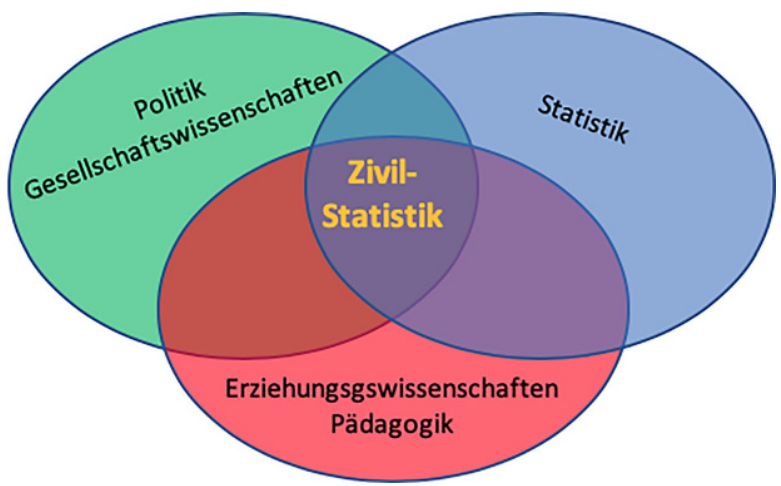

Wirtschaftsstatistik und der Statistical Literacy. Die folgenden Ausführungen werden deutlich machen, dass man die angestrebten Ziele und Inhalte nicht einfach mit schon bekannten begriffen belegen kann.

Jüngere staatliche Initiativen (siehe z.B. in den USA (https://data.gov), im UK (https://data.gov.uk) oder in Deutschland (https://govdata.de)) nennen ausdrücklich politische Ziele, insbesondere zur Förderung des demokratischen Prozesses, indem Bürgern Zugang zu Daten gewährt wird, die Debatten anregen und die Politikgestaltung beeinflussen können. Um die öffentliche Debatte auf Fakten statt auf Emotionen zu gründen und evidenzbasierte politische Entscheidungen zu fördern, muss die Statistikausbildung zwei Bereiche umfassen, die in der aktuellen Sekundarund Tertiärbildung vernachlässigt werden: das Verständnis multivariater Phänomene sowie das Denken mit und das Lernen aus komplexen Daten (Engel 2016, 2019). Zivilstatistik verlangt neben basalem mathematischem Wissen spezielle statistische Kenntnisse und liegt im Schnittfeld von Statistik, Gesellschaftswissenschaften (Gemeinschaftskunde, Politik etc.) und (aufgrund seines demokratischen Bildungsauftrags) Erziehungswissenschaft (siehe Abb. 1). Eine statistische Grundbildung oder Statistical Literacy ist eine Schlüsselkompetenz von Bürgern in Informationsgesellschaften und folglich auch als eine notwendige Komponente sowohl der Schul- wie der Erwachsenenbildung anzusehen: „Statistical Literacy is the ability to understand and critically evaluate statistical results that permeate our daily lives - coupled with the ability to appreciate the contributions that statistical thinking can make in public and private, professional and personal decisions“ (Wallman 1993, S. 1).

Zivilstatistik ist nicht einfach identisch mit Wirtschaft- und Sozialstatistik, und es geht auch nicht um die Anwendung (zumeist hochentwickelter) statistischer Methoden zur Beratung und Analyse in Politik und Wirtschaft, sondern für Zivilstatistik ist ein Bildungsauftrag zentral, der auf die Mitte der Gesellschaft abzielt: die Befähigung von informierten Bürgern zu evidenzbasierter Entscheidungsfindung und zivilgesellschaftlichem Engagement. Damit ist das, worum es bei Zivilstatistik geht, eine Weiterentwicklung und Implementierung von Statistical Literacy, die Elemente einer critical statistical literacy (Weiland 2017) aufgreift und mit modernen LehrLernkonzepten von Statistik (z.B. Ben Zvi and Garfield 2004) verbindet. Kontexte sind nicht mehr länger lediglich ein Vorwand für das Erlernen statistischer Verfahren, sondern stellen einen eigenständigen Lerngegenstand dar. Impliziert sind ge- 
sellschaftspolitische Reflexionen, die auf quantitativen Erkenntnissen beruhen. Sie sind der Schlüssel für die Herausbildung einer kritischen Bürgerschaft, in der die Lernenden nicht mehr Beobachter der Welt sind, sondern Teilnehmer und Transformatoren sozialer Krisen werden (Skovsmose 1994). Im EU-Projekt ProCivicStat haben wir den Begriff Zivilstatistik gewählt, um all die genannten Perspektiven konzise zusammenzufassen. Während Statistical Literacy als Basiskompetenz auf die allgemeine Fähigkeit abzielt (siehe z.B. Wallman 1993; Gal 2002), Statistiken aus allerlei Kontexten zu beurteilen, fokussiert Zivilstatistik auf Fragen von sozialer, gesellschaftlicher Relevanz. Auch schon frühere Ansätze in der Mathematikdidaktik von Fischer (1984), Borovcnik und Ossimitz (1987), Krüger (2012a, 2012b) oder der 1977 gegründete „Verein Mathematische Unterrichtseinheiten Datei MUED“ (www. mued.de) verknüpften Explorative Datenanalyse mit Zielen einer politischen Bildung und Emanzipation. Lesser (2007) bezeichnet Statistik als Grammatik der sozialen Gerechtigkeit und diskutiert, wie eine Reihe statistischer Konzepte im Kontext von Fragen der sozialen Gerechtigkeit behandelt werden können. Im Projekt ProCivicStat wurde ein theoretischer Rahmen für die Zivilstatistik entwickelt, es wurden spezifische Kompetenzen zum Verstehen von Statistiken über die Gesellschaft identifiziert und zahlreiche, konkrete und frei zugängliche Lehrmaterialien entwickelt, die unter Zugriff auf heutzutage frei verfügbare Datensätze und mächtige, didaktisch konzipierte Werkzeuge weitaus mehr Möglichkeiten zu tiefer gehenden Datenanalysen bieten als frühere Ansätze.

\subsection{Charakteristika zivilstatistischer Daten und darauf bezogener Publikationen}

Unsere Analysen aus dem Projekt ProCivicStat legen nahe, dass zivilstatistische Kompetenz besondere Merkmale aufweist, wie z.B. das Verständnis multivariater dynamischer (d.h. sich mit der Zeit auch kurzfristig ändernder) Phänomene in bedeutungsvollen sozialen Kontexten und das Verstehen von reichhaltigen Texten und Visualisierungen. Basierend auf diesen Analysen haben wir in ProCivicStat ein Modell entwickelt, das elf Wissensbasen und Prozesse beschreibt, die benötigt werden, um Statistiken über gesellschaftliche Phänomene zu verstehen, zu bewerten und mit ihnen zu argumentieren (ProCivicStat Partners 2018).

Statistiken über gesellschaftliche Phänomene haben oft spezielle Merkmale, die sich von Inhalten anderer Statistiken unterscheiden. Dazu gehören:

(i) Multivariate Daten zu komplexen Systemen mit Wechselwirkungen

Daten über interessante soziale Phänomene stehen meist nicht isoliert voneinander. Zivilstatistische Themen sind multivariater Natur; ihre Beschreibung und ihr Verständnis umfasst mehrere Variablen, die korreliert sind, miteinander interagieren oder nichtlineare Beziehungen untereinander haben. Dieser Punkt wurde auch von verschiedenen anderen Autoren auf unterschiedliche Weise betont; die Guidelines for Assessment and Instruction in Statistics Education der American Statistical Association (GAISE 2016) etwa bekräftigen die Tatsache, dass Phänomene in der modernen Welt ko-variieren, d.h. ein Phänomen ist beeinflusst oder wird verursacht von anderen Variablen oder Faktoren und interagiert mit oder beeinflusst wiederum andere Phänomene. Zivilstatistische Daten entstammen (im Gegensatz zu Daten aus 
Experimentalstudien) meist aus Beobachtungsstudien oder Erhebungen, weshalb eine belastbare Identifizierung von Ursache-Wirkungs-Zusammenhängen in der Regel sehr schwer ist.

(ii) Daten in verschiedenen Aggregationsstufen

Statistiken über gesellschaftliche Phänomene beinhalten oft Daten, die auf verschiedene Arten gruppiert (z. B. Männer vs. Frauen) oder auf Niveaustufen geordnet (z. B. Primar-, Sekundar- oder Tertiärbereich) sind. Darüber hinaus werden Daten oft für mehrere Untergruppen auf verschiedenen Aggregationsebenen berichtet. Mögliche Schlussfolgerungen können vom Aggregationsniveau beeinflusst werden, d.h. Muster in den Daten können sich ändern, wenn Daten innerhalb von Untergruppen aggregiert oder disaggregiert werden.

Statistische Ämter erstellen oft Schlüsselbotschaften an Entscheidungsträger und die breite Öffentlichkeit über die Höhe oder Veränderungen von Hunderten von Indikatoren wie Arbeitslosigkeit, Kindersterblichkeit oder Einkommensungleichheit. Solche Indikatoren können als einfache Raten berechnet oder als komplexe Aggregate von gewichteten Elementen (z.B. Verbraucherpreisindex, Gini-Koeffizient) abgeleitet werden. Sie können entweder auf objektiven (z.B. „Konsumausgaben“) oder subjektiven Daten (z.B. „Verbrauchervertrauen“) basieren, und ihre Definitionen, zugrundeliegenden Methoden oder Messmethoden können sich mit der Zeit entwickeln und verändern, um sich den Bedürfnissen der Gesellschaft anzupassen.

(iii) Zeitabhängige Daten und Notwendigkeit unterschiedliche Datenquellen zu beurteilen und zu integrieren

Amtliche Statistiken basieren häufig auf Daten, die regelmäßig (z.B. pro Monat, Quartal, Jahr) oder auf vergleichender Basis (z.B. in mehreren Ländern) erhoben werden, mitunter von verschiedenen Quellen und mit unterschiedlicher Methodik und unterschiedlicher Vergleichsbasis. Daten werden oft als Trend im Laufe der Zeit gemeldet und können aktualisiert werden, wenn neue Daten verfügbar oder alte Daten erneut ausgewertet werden.

Zivilstatistiken sind häufig nicht das Ergebnis einer einmaligen Datensammlung (im Gegensatz zu einer einzelnen Umfrage), sondern basieren auf mehreren Datenquellen. Dies können Daten sein, die periodisch mit der gleichen Methodik erhoben werden (z. B. eine Arbeitslosenumfrage jeden Monat oder jedes Jahr) oder auf vergleichender Basis (z.B. in mehreren Ländern) gemeldet werden. Folglich werden Daten häufig im Laufe der Zeit als Trend gemeldet und können aktualisiert werden, wenn neue Daten verfügbar oder alte Daten erneut ausgewertet werden. Darüber hinaus können verschiedene Interessensgruppen unterschiedliche Befunde und Meldungen zu den gleichen sozialen Phänomenen liefern (z. B. geschlechtsspezifisches Lohngefälle, Kriminalitätsraten zwischen verschiedenen sozialen Gruppen, Menschen unterhalb der Armutsgrenze) oder unterschiedliche Indikatoren oder Aggregationsebenen verwenden, um ihre sozialen Phänomene zu analysieren und Ergebnisse zu kommunizieren.

(iv) Vielfältige nicht-lineare und interaktive Texte mit unterschiedlicher Darstellungstiefe von Daten, wissenschaftlichen Fragen und Ergebnissen, die zusätzliche Recherchekompetenz zur Interpretation erfordern

Statistische Informationen über die Gesellschaft werden der Öffentlichkeit hauptsächlich von statistischen Ämtern über veröffentlichte Texte (z. B. Pressemitteilun- 
gen, Kurzberichte), über Artikel und Werbetexte in Printmedien oder digitalen Medien oder als Teil von gesprochenen Präsentationen wie in TV-Nachrichtenprogrammen zugänglich gemacht und bestehen oft aus einer Kombination von grafischen, tabellarischen und textuellen Elementen, die man in der Didaktik auch als nicht-lineare oder diskontinuierliche Texte bezeichnet. Text ist ein primäres Medium für die Kommunikation von Statistiken, und die Öffentlichkeit muss verschiedene Arten des Schreibens verstehen, z.B. die formalisierte Sprache, die oft in offiziellen Berichten verwendet wird, journalistische Texte und mehr. Texte auf Webseiten enthalten zunehmend interaktive Elemente, interaktive Graphiken und Links.

Zivilstatistiken werden häufig durch journalistische Berichterstattung in den Printund visuellen Medien oder über private Einrichtungen wie Nichtregierungsorganisationen, Interessengruppen usw. an die Öffentlichkeit vermittelt. Diese „sekundären Akteure "wählen in der Regel nur ausgewählte Aspekte der Originalpublikationen aus, und manchmal präsentieren sie diese in einer Weise, die bestimmte Ansichten fördert. Dies erfordert daher von Leserinnen und Lesern eine kritische Interpretation.

Ein besonderes Merkmal solcher Texte ist ihre Anreicherung mit Daten, Auswertungen und Visualisierungen (Nicht-Linearität), die besondere „Lesekompetenz“ erfordert, ggf. auch Recherchekompetenz, indem man ausgehend von einem zusammenfassenden Text nach den Originalquellen oder Artikeln mit unterschiedlichen Verarbeitungsgraden der Originalarbeiten sucht, um den Hintergrund für die Interpretation der Texte zu verbreitern. In jüngster Zeit hat sich eine eigene Profession von „Data Journalism“ herausgebildet, die in umfassender Weise interaktive Technologien zur Datenaufbereitung und -visualisierung bei journalistischen Beiträgen nutzen (Loos 2018).

\section{(v) Innovative (interaktive) Visualisierungen}

Daten zu gesellschaftlichen Problemen werden heute oft mit reichhaltigen, neuartigen Datenvisualisierungen kommuniziert. Nutzer benötigen Fähigkeiten zum Verständnis von Daten, die auf neuartige Weise präsentiert werden, und sie benötigen Fähigkeiten zum Zugreifen auf und Manipulieren von computervermittelten interaktiven Visualisierungen, die sich auf vielen Websites finden. Da Daten und Erkenntnisse über soziale Phänomene (wie Höhe oder Veränderungen von Unfallraten, Einkommen, Arbeitslosigkeit, Armut, usw.) multivariat, dynamisch und aggregiert sind, erfordert ihre Beschreibung über die Zeit oder andere Vergleichseinheiten (z. B. Regionen, soziale Gruppen) die Verwendung von verschiedenen Arten von Darstellungen. Daher begegnen Benutzer und Leser heutzutage einer Reihe von statischen, dynamischen und interaktiven Visualisierungen (Ridgway 2015) oder Infografiken, die im Vergleich zu dem begrenzten Bereich von Graphen, Histogrammen und Tabellen, die in einführenden Statistikklassen verwendet werden, viel breiter und ausgeklügelter sind.

Nicht alle diese Merkmale sind in allen Zivilstatistiken präsent. In ihrer Kombination stellen sie jedoch einzigartige Anforderungen dar. Nachstehend finden sich zwei Beispiele, die veranschaulichen sollen, wie sich grundlegende Merkmale von Zivilstatistik in verschiedenen Arten von Botschaften manifestieren. Das erste Beispiel bezieht sich auf eine (statistische) Veröffentlichung eines statistischen Amtes, das zweite auf die Nutzung eines interaktiven web-basierten Tools. 


\subsubsection{Beispiel: Armutsrisiko}

Am 8. November 2017 veröffentlichte das Statistische Bundesamt in Wiesbaden eine Pressemitteilung (Nr. 3923) bezüglich des Risikos von Armut und sozialer Ausgrenzung in Deutschland:

\section{„19,7 \% der Bevölkerung Deutschlands von Armut oder sozialer Ausgren- zung bedroht Anteil EU-weit bei 23,5 \%}

WIESBADEN - Während der Anteil der von Armut oder sozialer Ausgrenzung bedrohten Bevölkerung in der Europäischen Union (EU) im Jahr 2016 durchschnittlich bei 23,5\% lag, betrug dieser Anteil in Deutschland 19,7\%. Wie das Statistische Bundesamt (Destatis) nach den Ergebnissen der Erhebung LEBEN IN EUROPA (EU-SILC) weiter mitteilt, waren in Deutschland 16 Mio. Menschen von Armut oder sozialer Ausgrenzung betroffen.

Eine Person gilt als von Armut oder sozialer Ausgrenzung bedroht, wenn mindestens eine der folgenden drei Lebenssituationen zutrifft: Ihr Einkommen liegt unter der Armutsgefährdungsgrenze, ihr Haushalt ist von erheblicher materieller Entbehrung betroffen oder sie lebt in einem Haushalt mit sehr geringer Erwerbsbeteiligung. Für jede dieser Lebenssituationen kann jeweils der Anteil der Personen in der Bevölkerung ermittelt werden, auf den diese Situation zutrifft:

- Mit 16,5\% der Bevölkerung war jede sechste Person in Deutschland im Jahr 2016 armutsgefährdet. Das entsprach rund 13,4 Mio. Menschen. Eine Person gilt nach der EU-Definition für EU-SILC als armutsgefährdet, wenn sie über weniger als $60 \%$ des mittleren Einkommens der Gesamtbevölkerung verfügt (Schwellenwert der Armutsgefährdung). 2016 lag dieser Schwellenwert für eine alleinlebende Person in Deutschland bei $1064 €$ im Monat, für zwei Erwachsene mit zwei Kindern unter 14 Jahren bei $2234 €$ im Monat.

- 3,7\% der Bevölkerung in Deutschland waren im Jahr 2016 von erheblicher materieller Entbehrung betroffen. Das bedeutet, dass ihre Lebensbedingungen aufgrund von fehlenden finanziellen Mitteln eingeschränkt waren. Sie waren zum Beispiel nicht in der Lage, ihre Rechnungen für Miete, Hypotheken oder Versorgungsleistungen zu bezahlen, ihre Wohnungen angemessen $\mathrm{zu}$ beheizen oder eine einwöchige Urlaubsreise zu finanzieren.

- 9,6\% der Bevölkerung unter 60 Jahren lebten in einem Haushalt mit sehr niedriger Erwerbsbeteiligung und damit in Haushalten, in denen die tatsächliche Erwerbsbeteiligung der erwerbsfähigen Haushaltsmitglieder im Alter von 18 bis 59 Jahren insgesamt weniger als $20 \%$ betrug.

Im EU-Durchschnitt waren 17,3\% der Bevölkerung von Armut bedroht sowie $7,5 \%$ von erheblicher materieller Entbehrung betroffen. 10,4\% lebten in einem Haushalt mit sehr niedriger Erwerbsbeteiligung. “

Die Erklärung zeigt einige der oben aufgeführten Charakteristika von Zivilstatistik. Armut und soziale Ausgrenzung sind multivariate Phänomene, da mehrere Faktoren Einfluss darauf haben. Das Ausmaß der Armut kann nicht beschrieben

\footnotetext{
3 https://www.destatis.de/DE/PresseService/Presse/Pressemitteilungen/2017/11/PD17_392_634.html.
} 
oder verstanden werden, ohne zusätzliche Variable wie Alter oder Anzahl der Kinder in einem Haushalt einzubeziehen. Die monatlichen Erhebungen der Armutsrate, immer auch mit dem Verweis auf den Vormonat oder das Jahr, verdeutlichen den dynamischen Charakter der Daten. Im Text wird zwar kurz erklärt, was Armut und soziale Ausgrenzung bedeuten, allerdings sind die Begriffe materielle Entbehrung und mittleres Einkommen (was die Armutsgefährdung definiert) anhand der Pressemitteilung nicht eindeutig.

Nähere Informationen kann man von der Website des Statistischen Bundesamtes erhalten: Materielle Entbehrung liegt dann vor, wenn nach Selbsteinschätzung 4 von 9 Kriterien erfüllt sind. Wer hat diese Kriterien ausgewählt und wie aussagekräftig sind Selbsteinschätzungen?

Ist mit dem mittleren Einkommen der Median oder das arithmetische Mittel gemeint? - das hat deutliche Auswirkungen darauf, wer unterhalb des 60\%-Wertes liegt. Zudem haben der Wohnort und die damit verbundenen Lebenshaltungskosten auch einen großen Einfluss auf eine mögliche Armutsgefährdung oder soziale Ausgrenzung. Eine detaillierte Beschreibung mittels disaggregierter Daten - wer besonders von Armut gefährdet ist - wäre in einem nächsten Schritt denkbar und aufschlussreicher als eine globale Aussage über 16,5\% der gesamten Bevölkerung, abgesehen davon, dass aus dem Text nicht hervorgeht, in welcher Beziehung die verschiedenen Prozentsätze für Armutsgefährdung bzw. Armutsgefährdung und sozialer Ausgrenzung zueinander stehen.

\subsubsection{Beispiel: Lebenserwartung und Einkommen}

Das folgende Beispiel zeigt, wie man mit dem Onlinetool Gapminder (www. gapminder.org) multivariate zivilstatistische Daten (zum größten Teil Daten aus UNErhebungen) visualisieren und explorieren kann. Dabei enthalten die Daten viele der oben formulierten Besonderheiten zivilstatistischer Daten wie z. B. multivariate Daten zu komplexen Systemen mit Wechselwirkungen, Daten in verschiedenen Aggregationsstufen oder innovative Visualisierungen. Viele dieser Daten sind in einem größeren Zeitraum (z.B. von 1800-2018) erhoben worden und ermöglichen daher auch die Untersuchung der Entwicklung von Zusammenhängen im Laufe der Zeit. Insbesondere lassen sich so verschiedene Zusammenhänge zwischen Variablen untersuchen, wie z. B.: Gibt es einen Zusammenhang zwischen der Lebenserwartung, die ein gerade geborenes Kind nach den aktuellen Bedingungen hat und dem Bruttoinlandsprodukt pro Person (bereinigt um Kaufkraftunterschiede)? In Abb. 2 ist ein Gapminder-Diagramm der beiden Variablen Lebenserwartung bei Geburt (Life expectancy in Jahren) und Einkommen (Kaufkraftbereinigtes Bruttoinlandsprodukt pro Person in internationalen Dollar, Festpreise 2011) dargestellt. Im Falle der Exploration des oben genannten Zusammenhangs kann es sich erst einmal anbieten, den Zusammenhang zwischen Lebenserwartung und Pro-Kopf-Einkommen in der Standard-Einstellung (Jahr 2018, nach Anklicken des Links) zu betrachten.

Neben den Variablen Einkommen und Lebenserwartung sind in diesem Beispiel auch die Variablen Einwohnerzahl des Landes und zugehöriger Kontinent in die Visualisierung einbezogen. Zu beachten ist allerdings, dass die X-Achse mit dem Merkmal Einkommen nicht linear sondern logarithmisch skaliert ist. Das heißt der lineare 
Abb. 2 Graphische Darstellung der Variablen Lebenserwartung (Life expectancy) und Einkommen (Income per person, auf einer logarithmischen Skala); jede Blase repräsentiert ein Land für die Situation im Jahr 2018. Die Fläche der Blasen ist proportional zur Einwohnerzahl des jeweiligen Landes während die Farbe den Kontinent repräsentiert. (Quelle: freies Material von www.gapminder.org)

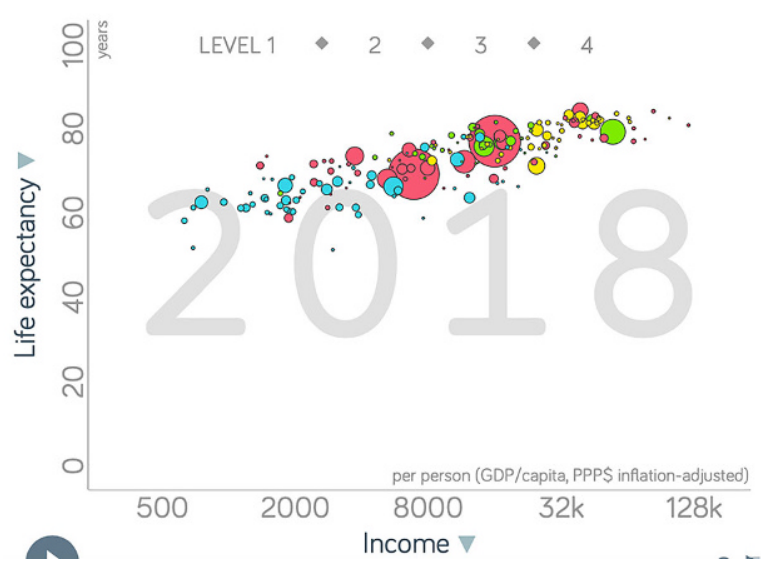

Zusammenhang muss so interpretiert werden, dass eine jeweilige Verdoppelung des Pro-Kopf-Einkommens jeweils zum gleichen additiven Zuwachs an Lebenserwartung führt. Für viele Bürger stellen solche Skalen, die nicht (mehr) Gegenstand des Schulstoffes sind, bereits schwer zu überwindende Hürden dar.

Gapminder bietet auch die Möglichkeit, die entsprechende Situation im jeweiligen Land genauer zu erkunden. Durch Anklicken der einzelnen Blasen können die zeitliche Entwicklung von Lebenserwartung und Pro-Kopf-Einkommen in jedem Land der Welt, von dem Daten in Gapminder vorliegen, betrachtet werden. Klickt man auf den „Playbutton“ links unterhalb der Grafik startet eine „Zeitreise“ ab dem Jahr 1800, die die Entwicklung des Zusammenhangs zwischen Einkommen und Lebenserwartung im Laufe der Zeit visualisiert. Somit werden in einer Darstellung fünf Variable repräsentiert (wobei die Variable „Zeit““ nur Online per Knopfdruck animiert und nicht in der statischen Darstellung von Abb. 2 sichtbar wird. Dazu ist ein Besuch auf der Website www.gapminder.org nötig).

\section{Ein konzeptioneller Rahmen für zivilstatistische Kompetenzen}

Die Beschreibung der fünf Hauptmerkmale von zivilstatistischen Daten und die Beispiele implizieren, dass Zivilstatistiken reichhaltige multivariate Informationen beinhalten, die Texte (z. B. Pressemitteilungen, Artikel in den Medien) mit Hintergrundinformationen, evidenzbasierten Behauptungen und statistischen Informationen kombinieren, die über visuelle Elemente (Grafiken, Videos) und zugrunde liegende Daten und Datensätze übermittelt werden.

Aus den obigen Beispielen wird deutlich, dass ein kompetenter Umgang mit gesellschaftlich relevanten Daten interdisziplinäre Anforderungen stellt, spezielle Wissenselemente und Grundfertigkeiten beinhaltet und ebenso affektive bzw. dispositionale Elemente umfasst. Im Zentrum, als Ausgangs- und Endpunkt jeder Beschäftigung mit Zivilstatistik, steht die kritische Bewertung und Reflektion eines gesellschaftlich bedeutsamen Themenbereichs. Offensichtlich ist allgemeines und spezielles statistisches Wissen, z.B. zu Darstellungen und Modellen und zur Me- 
thodik der Datenerhebung ebenso nötig wie kontextbezogenes soziales, sozio-ökonomisches oder gesellschaftliches Wissen. Um vorhandenes spezielles Wissen und Interesse zu zivilstatistischen Themen aktivieren oder akquirieren zu können, bedarf es gegebenenfalls weiterhin bestimmter Fertigkeiten bei der Datenrecherche und bei der Aufbereitung und Säuberung von Daten zur Erkundung mit passender Soft- und Hardware. Außerdem sind basisnumerische Fähigkeiten (z. B. Verstehen von Prozenten, angemessene Vorstellungen von zahlenmäßigen Größenordnungen) ebenso grundlegend wie ein angemessenes Lese- und Textverständnis sowie kommunikative Fähigkeiten, um erarbeitete Positionen und Schlussfolgerungen angemessen kommunizieren zu können (Engel 2019).

Nicholson et al. (2018) haben in Kooperation mit den Partnern im PCS-Projekt ein Modell entwickelt, das elf Facetten umfasst und prinzipiell wünschenswerte Fähigkeiten und Einstellungen im Bereich Zivilstatistik beschreibt. Das Modell ist in drei Gruppen oder Dimensionen organisiert, zu denen bestimmte Unterkategorien gehören (siehe Abb. 3):

Engagement und Aktion: Bereitschaft zum gesellschaftlichen Engagement; kritische Bewertung und Reflexion; Einstellungen

Wissen: Statistik und Risiko; Modelle und Darstellungen; Methodik und Forschungsprozess; Erweiterungen im Bereich amtlicher Statistik; kontextbezogenes gesellschaftliches Wissen

Prozessbezogene Kompetenzen: Informations- und kommunikationstechnologische Kompetenzen und Kompetenzen zur Informationsrecherche; Basiskompetenzen im Umgang mit quantitativen Informationen (z.B. Prozente etc.); Lese- und Textverständnis, basale Kommunikationsfähigkeiten

Dies sind insgesamt sehr anspruchsvolle ideale Forderungen, die einen Orientierungsrahmen abstecken. Im jeweiligen Bildungskontext ist $\mathrm{zu}$ entscheiden, was in welchem Umfang davon umgesetzt werden kann. Für die Sekundarstufen wären in diesem Rahmen Schülerinnen und Schüler zu befähigen, nicht primär zivilstatistische Analysen im Detail durchführen zu können, sondern kommunikationsfähig mit Experten zu werden, so wie es zum Beispiel höhere Allgemeinbildungskonzepte von Fischer (2001) und Konzepte der Civic Education von Vohns $(2016,2017)$ ausdrücken.

Die folgende Darstellung ist im Wesentlichen eine ins Deutsche übersetzte Kurzfassung von Nicholson et al. (2018). Für tiefergehende Erläuterungen und Begründungen verweisen wir auf die Originalarbeit.

1. Facette: Bewusstseinsmäßige und wissensmäßige Voraussetzungen für eine $B e$ reitschaft zum gesellschaftlichen Engagement

Diese Facette steht im Zentrum von Zivilstatistik. Sie liefert die Rechtfertigung dafür, warum überhaupt Daten benötigt werden. Gesellschaften benötigen Informationen über ihren aktuellen Stand, über Trends und Veränderungen, von der Vergangenheit in die Gegenwart und für Prognosen in die Zukunft. Daher benötigen sich engagierende Staatsbürger ein Bewusstsein und Wissen darüber, dass 


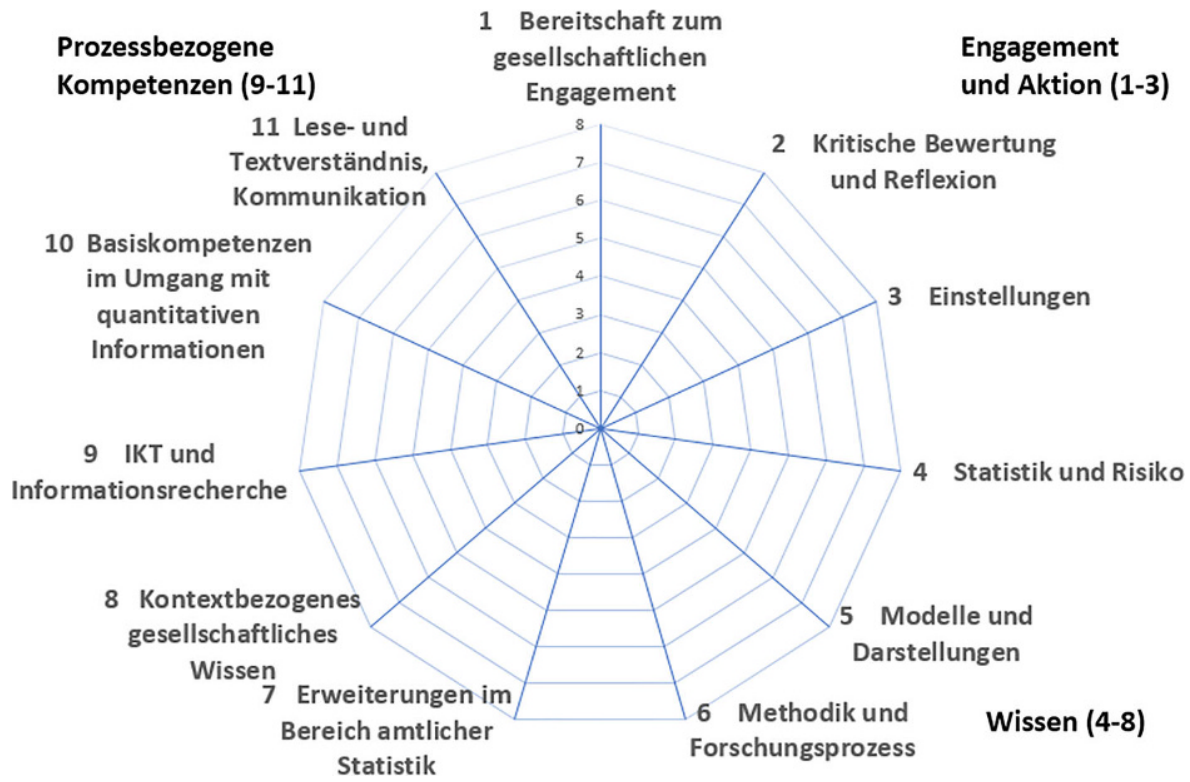

Abb. 3 Ein konzeptioneller Rahmen für Zivilstatistik

- es in der Gesellschaft „brennende“ soziale Probleme gibt, z. B. in Bezug auf Beschäftigung, Löhne, Kriminalität, Umweltverschmutzung, wirtschaftliche Chancen und Chancengleichheit, Zugang zu Dienstleistungen usw.,

- es zu diesen Themen sozialpolitische Entscheidungen und Programme gibt,

- Politik von Politikern und Entscheidungsträgern geprägt wird - und Entscheidungen, Wahlen und Risiken erfordern, bei denen vorhandene Möglichkeiten, Optionen und deren Wahrscheinlichkeiten, Kosten und Nutzen, erwartete Werte und subjektive Nutzen abgewogen werden,

- Daten, Statistiken und Fakten die sozialen Programme beeinflussen können und es Qualitätsniveaus für „Evidenzen“ gibt,

- die Bürger die Politikgestaltung beeinflussen können,

- sie sich befähigt fühlen, sich gesellschaftlich zu engagieren.

\section{Facette: Kritische Bewertung \& Reflexion}

,Postfaktische“ Argumente und ,,alternative Fakten“ sind Attacken auf das Kerngebiet informierter Entscheidungsfindung. Auch bei der Verwendung von Daten aus gut authentifizierten Quellen besteht noch die Notwendigkeit kritischer Bewertung und Reflexion. So sollte sich ein zivilstatistisch gebildeter Bürger z. B. routinemäßig folgende Fragen stellen, wenn er eine datenhaltige Veröffentlichung liest:

- Welche Geschichte wird hier mit den Daten erzählt - wessen Geschichte und warum wird sie erzählt? Wessen Sicht wird hierbei eingenommen?

- Ist die Sachlage angemessen benannt worden? 
- Welcher Nachweis wird geliefert? Von einer glaubwürdigen Quelle?

- Wurden angemessene statistische Modelle verwendet? Ist es z. B. in Ordnung anzunehmen, dass die Daten normalverteilt sind? Ist Linearität (zu Recht) angenommen worden?

- Worum geht es hier sonst noch - gibt es erklärende Drittvariable (Confounder)?

- Sind die Schlussfolgerungen konsistent mit der vorgelegten Evidenz?

\section{Facette: Einstellungen}

Einstellungen spiegeln ein komplexes Netz aus Werten, Motivationen, Überzeugungen und Haltungen wider. Sie haben eine soziale Dimension wie die Bereitschaft, Interpretationen mit anderen zu teilen. Sie haben eine ethische Dimension (,Warum geht mich das Ganze überhaupt etwas an?"). Sie haben Komponenten, die das eigene Engagement sowohl in positiver als auch in negativer Weise beeinflussen (z.B. Selbstwirksamkeit, Vertrauen). Das Ignorieren von Evidenz aufgrund von Überzeugungen, die unkritische Akzeptanz neuer Informationen oder der Glaube, dass soziale Phänomene nur von Experten verstanden werden können, sind alles Symptome von problematischen Einstellungen.

\section{Facette: Statistik und Risiko}

Diese Facette enthält viel von dem, was gemeinhin in einführenden universitären Statistikkursen gelehrt wird: Stichproben, Populationen und Repräsentativität; Variabilität; Beschreiben und Vergleichen von Verteilungen; Assoziation und Korrelation; Regression; Nichtlinearität; Signal und Rauschen; Interaktion; Bayesianisches Schließen; Schätzen; Effektgröße. Das Einschätzen von Risiken ist ein wichtiger Bestandteil von Zivilstatistik. Es beruht auf den Konzepten von Wahrscheinlichkeit und bedingter Wahrscheinlichkeit (einschließlich des Satzes von Bayes), des Erwartungswerts und (der Quantifizierung von) subjektivem Nutzen. Zivilstatistik erfordert auch ein Verständnis für einige der Ideen rund um Big Data - wie die Vertrautheit mit einer Vielzahl von Datenquellen und zugehörigen Techniken der Analyse, vor allem derjenigen, die für die Erkennung von Mustern verwendet werden.

\section{Facette: Modelle und Darstellungen}

Im Zentrum von Statistik steht die Anwendung mathematischer Modelle auf relevante Anwendungen. Ein wesentlicher Bestandteil von zivilstatistischer Kompetenz besteht darin zu verstehen, dass bei der Modellierung komplexer sozialer Phänomene qualitativ verschiedene Modelle verwendet werden können, um ein und dasselbe Phänomen zu modellieren. Zum Beispiel könnten ein Ökonom und ein Soziologe ganz unterschiedliche Methoden anwenden, um „Armut“ zu studieren und ganz unterschiedliche Theorien der Verursachung von Armut haben. Zivilstatistik erfordert die Fähigkeit, den Modellcharakter von Analysen und Vorhersagen zu erkennen und zu verstehen sowie die grundlegenden Annahmen eines Modells in Frage stellen zu können.

Darstellungen sind eine Kernkompetenz zum Verstehen von Phänomenen. Zivilstatistik erfordert Vertrautheit mit anspruchsvollen Darstellungen und Visuali- 
sierungen einschließlich solcher, die dynamisch und interaktiv sind. In jüngster Zeit gibt es eine Explosion in der Verwendung technologie-basierter Darstellungen und Visualisierungen. Zivilstatistik erfordert die Fähigkeit, diese zu verstehen und innovative Darstellungen kritisch zu bewerten.

\section{Facette: Methodik und Forschungsprozess}

Zivilstatistik erfordert idealerweise ein Verständnis von Stärken und Schwächen verschiedener Forschungsmethoden und einige verfahrenstechnische Fähigkeiten. Ein Verständnis ethischer Fragen im Zusammenhang mit der Datenerzeugung und dem Einsatz verschiedener Forschungsmethoden ist ebenso wesentlicher Bestandteil der Zivilstatistik wie etwa die Notwendigkeit, sich über Fragen der Vertraulichkeit und des Schutzes der Identität der Bürger zu informieren.

7. Facette: Erweiterungen der statistischen Kompetenz im Hinblick auf wichtige Aspekte amtlicher Statistik

Produzenten amtlicher Statistik (Statistische Ämter, EuroStat, UN etc.) sind eine wichtige Quelle für datenbasierte Evidenz bezüglich gesellschaftlich relevanter Fragen. Viele der zentralen Ideen, die sie verwenden, erhalten wenig Aufmerksamkeit in traditionellen Statistikkursen wie zum Beispiel: Umfrage-Design (und damit verbundene Probleme wie Nicht-Antwort oder Antwortverzerrungen); Messprobleme (Zuverlässigkeit und Gültigkeit, Metadatendefinitionen); Operationalisierung von Variablen sowie Definition und Bedeutung von Indizes; Techniken wie gleitende Mittelwerte, Datenglättung, saisonale Anpassung und Fallgewichtung; synthetische Methoden, bei denen Daten, die durch konventionelle Umfragedaten gesammelt wurden, mit Big Data (z.B. Daten über Mobilfunkverkehr oder Websuchdaten) kombiniert werden.

\section{Facette: Kontextbezogenes gesellschaftliches Wissen}

In der Statistik geht es um Modellierungen, und um zu modellieren muss man zumindest ein grundlegendes Verständnis der Phänomene haben, die modelliert werden sollen. Manches davon kann reine Sachkenntnis sein - etwa, zu wissen, dass die Aufnahme von einer Millionen Flüchtlinge in Deutschland (etwa 82 Mio. Einwohner) leichter sein dürfte als eine Millionen Flüchtlinge in Österreich aufzunehmen (etwa 8,5 Mio. Einwohner), wenn alles andere im Wesentlichen gleich ist.

$\mathrm{Zu}$ wissen, dass andere Dinge sicher nicht gleich sind, erfordert ein tieferes kontextuelles Wissen. Kontextbezogene gesellschaftliche Kenntnisse umfassen z. B. Allgemeinwissen wie Größe der Bevölkerung, Größe des Bruttosozialproduktes, Staatsverschuldung und Ressourcen; Demographie; Geschichte und Geographie; Regional- und Geopolitik. Ein Vorteil des kontextuellen gesellschaftlichen Wissens ist, dass man anhand von Kenntnissen plausibler Kovariablen nach alternativen Erklärungen und Analysen suchen kann.

\section{Facette: Informations- und Kommunikationstechnologie und Informationsrecher- che}

Viele offizielle Statistikämter (z. B. Statistisches Bundesamt, EuroStat, UN) machen Daten öffentlich zugänglich - aber der Zugriff und die direkte Bearbeitung 
dieser Datensätze erfordern oftmals ein beträchtliches Fachwissen und informationstechnische Kenntnisse.

Big Data ist eine weitere wichtige Informationsquelle für Zivilstatistik. Beispiele hierfür sind Daten aus mobilen Endgeräten, Transaktionsdaten von Mobiltelefonen und Daten, die von Webseiten extrahiert (webscraping) wurden. Zivilstatistik erfordert ein Verständnis von analytischen Techniken, die für den Zugriff und die Analyse von multivariaten und unstrukturierten Daten geeignet sind.

IKT-Kenntnisse sind erforderlich, um mit IKT-basierten Tools wie Statistikpaketen zu arbeiten. Für Zivilstatistik müssen ist es erforderlich, interaktive Displays effektiv zu nutzen.

\section{Facette: Basiskompetenzen im Umgang mit quantitativen Informationen}

Quantitative Fähigkeiten sind die Grundlage aller Aspekte von Statistical Literacy. Zu den Komponenten gehören ein Verständnis für Zahlen (number sense), Verhältnisse, Prozentsätze, Raten und Brüche. In Zivilstatistik sind sehr große Zahlen üblich, und scheinbar große Ressourcen können tatsächlich im Kontext klein sein. Es ist leicht, Beispiele zu finden, bei denen ein Autor bewusst (akkurate) Daten gewählt hat, um in die Irre zu führen - zum Beispiel, wenn eine prozentuale Zunahme berichtet wird, wobei die Zunahme des Ausgangswertes in absoluten Zahlen gering ist.

\section{Facette: Lese- und Textverständnis und Kommunikation}

Vielfach werden Informationen auch als Text, Diagramm oder Bild in gedruckter Form präsentiert. Solche Texte sind oft sehr dicht. Es ist dann eine wesentliche Fähigkeit, den Text zu verstehen, mit gezeigten Darstellungen in Verbindung zu bringen und den Gesamtsinn eines Artikels aufzunehmen.

Allerdings stellen sowohl notwendiges Lese- und Textverständnis als auch Kommunikationsfähigkeit Ziele dar, die sich in ständiger Veränderung und Anpassung befinden. Neue Formen der Kommunikation entstehen, die soziale Medien und neue Wege zur Visualisierung von Daten und Videos einschließen. Bürgerinnen und Bürger müssen in der Lage sein, die in diesen neuen Kommunikationsformen vermittelten Nachrichten zu verstehen und zu dekonstruieren. Für ein Engagement im Bereich Zivilstatistik müssen Bürgerinnen und Bürger auch in der Lage sein, auf neue Weise zu kommunizieren.

\section{Herausforderungen und Entwicklungen jenseits von Schule und Hochschule}

Statistische Kenntnisse der Öffentlichkeit gezielt zu verbessern ist nicht nur ein genuines Interesse von Lehrkräften an Schule und Hochschule. Nationale und internationale Statistikämter sowie Organisationen, die sich mit dem Wohl der Öffentlichkeit befassen (z. B. im Gesundheitsbereich oder bei Wohlfahrtsverbänden) benötigen als Produzenten, aber auch als Vermittler und Interpreten quantitativer Informationen Personal, das zivilstatistische Inhalte verstehen und verständlich vermitteln kann. Das gilt in zunehmendem Maße vor allem auch für Journalisten und unabhängige Or- 
ganisationen zur Überprüfung von Fakten, sogenannte fact-checkers. Einerseits sind diese Berufsgruppen herausgefordert, ihre Informationen in verständlicher Sprache und mit geeigneten Visualisierungen zu präsentieren. Andererseits muss die breite Öffentlichkeit in die Lage versetzt werden, Sinn aus diesen Produkten zu erschlieBen, um sich als mündige Bürger informiert in öffentliche Entscheidungsprozesse einbringen zu können.

Statistische Ämter sind daran interessiert, die Nutzung ihrer Informationsprodukte durch mehrere Benutzergruppen, zu denen auch die breite Öffentlichkeit gehört, zu steigern. Sie unternehmen viele Schritte, um die Qualität ihrer Informationsdienste zu verbessern. Sie haben den freien Zugang zu ihren Informationsprodukten über digitale Portale eröffnet und suchen nach Wegen, um das Vertrauen der Öffentlichkeit in die amtlichen Statistiken und die Zufriedenheit mit ihren Informationsprodukten zu verbessern (de Smedt 2016), z.B. durch erklärende Videos und Dossiers zu einzelnen Themen. Selbst wenn offizielle Statistik-Websites benutzerfreundlicher gestaltet werden, ist das Verständnis der darin enthaltenen Informationen für den sich informierenden Bürger oft noch immer schwer (Schield 2011). Bisher wurde weitgehend auf die Bereitstellung von Schulungen oder Ressourcen im Zusammenhang mit amtlichen Statistiken für ein breiteres, nichtprofessionelles Publikum verzichtet. Nur sehr wenige offizielle Statistikanbieter bieten strukturierte Materialien an, mit denen die Öffentlichkeit oder Akteure aus dem Bildungssektor (d.h. Lehrer und Schüler) die offiziellen Statistiken auf ihren Websites besser verstehen können. Um die statistischen Kenntnisse der Öffentlichkeit zu verbessern, kann ein Ansatz darin bestehen, neben den reinen Daten auch vermehrt Erklärungen zu methodischen Fragen der Datenerhebung sowie interpretative Kommentare aufzunehmen. Eine andere Möglichkeit besteht darin, Links zu Tutorials bereitzustellen, die zentrale Grundbegriffe erklären.

Eine zentrale Rolle in der Vermittlung von Informationen mit statistischem Inhalt zu gesellschaftlich wichtigen Themen nehmen Journalisten ein. Eine noch relativ neue Entwicklung des Informationszeitalters und des Fortschritts digitaler Medien ist der Aufstieg des Datenjournalismus. Zunehmend stellen Printmedien auf ihren Internetseiten dynamische Visualisierungen bereit, oftmals Unikate für eine Thematik, die den Lesern individuell spezifische Detailfragen erkunden lassen (siehe z. B. http://www.theguardian.com/data oder http://www.zeit.de/datenjournalismus).

Datenjournalismus ist eine qualitativ neue Art der Berichterstattung, die Einblicke in relevante gesellschaftliche Trends gibt, indem offene Datensätze mit (halb-) automatisierten Methoden analysiert werden, um aussagekräftige Muster in der Datenstruktur zu erkennen (Rinsdorf 2016). Gemäß der Open-Data-Idee bedeutet Datenjournalismus nicht nur die Recherche in Datenbanken, sondern die Sammlung, Aufbereitung, Analyse und Publikation öffentlich zugänglicher Informationen sowie ihre Verarbeitung in klassischen journalistischen Darstellungsformen für die breite Öffentlichkeit. Dies verlangt von Journalisten völlig neue Fähigkeiten und Zugang zu neuen Ressourcen.

Mittels sozialer Medien ist es heutzutage viel einfacher, falsche Nachrichten zu verbreiten oder sie als alternative Fakten zu verbergen. Das Internet ist aber nicht nur ein Instrument zur Verbreitung von zweifelhaften oder bewusst gefälschten Informationen. Es ist zugleich auch ein Werkzeug, das durch den Zugang der Öffentlichkeit 
zu frei verfügbaren Daten und Regierungsdokumenten Möglichkeiten bereitstellt, Behauptungen, die im öffentlichen Diskurs propagiert wurden, zu überprüfen. Hier kommt überparteilichen und unabhängigen Fakten-prüfenden Organisationen mit ihren sorgfältigen Analysen und Überprüfungen eine ganz zentrale Kontrollfunktion zu. Zur Illustration zeigen wir drei Aussagen, die von PolitiFact (http://www. politifact.com) diskutiert und überprüft wurden:

- „Die 62 reichsten Menschen der Welt besitzen dasselbe Vermögen wie die 3,6 Mrd. Ärmsten“ (Oxfam USA am 07.10.2016), https://www.politifact.com/ personalities/oxfam-america/

- „Deutschland ist mit Kriminalität übersät“ (Donald Trump in einem TV Interview am 12. August 2016), https://www.politifact.com/truth-o-meter/statements/2016/ may/11/donald-trump/donald-trump-says-germany-now-riddled-crime-thanks/

- „Illegale Grenzübertritte an der Südgrenze [der USA] sind $40 \%$ reduziert““ (Mike Pence, 25. April 2018), https://www.politifact.com/wisconsin/statements/ 2018/may/11/mike-pence/mike-pence-uses-outdated-figure-claiming-large-dro/

Die jeweiligen Aussagen inklusive ihrer Quellen sind zunächst auf den genannten Webseiten dokumentiert. Es folgen dann Analysen mit Bezug zu Daten von vertrauenswürdigen Quellen und eine schlussfolgernde Einschätzung des Wahrheitsgehaltes der Aussagen.

Es ist nicht der Zweck dieses Beitrags, die Korrektheit dieser Behauptungen zu erörtern. Der interessierte Leser und die interessierte Leserin mögen die Details auf den obigen Websites untersuchen, die einige statistische Argumente und Verweise auf offene Datenquellen enthalten. Wir halten fest, dass Überprüfungen von politischen Aussagen möglich und Analysen von unabhängigen Organisationen im Internet verfügbar sind und ein Verständnis dieser Analysen zivilstatistische Kompetenzen verlangt.

Neben dem Überprüfen von Aussagen stellt die britische Organisation Full Fact (https://fullfact.org) zusätzlich einen Werkzeugkasten zur Verfügung, der der Öffentlichkeit helfen soll, Fake News als solche zu enttarnen. Im Anhang listen wir einige dieser Organisationen auf, die es sich zur Aufgabe gemacht haben, mittels Datenanalysen Behauptungen aus dem politischen Kontext jeweils landesspezifisch oder bezogen auf einen Kontinent zu prüfen.

\section{Beispiele, Lehr- und Lernmaterialien}

\section{1 Überblick}

Die in Abschn. 3 vorgestellten Kompetenzen geben einen umfassenden Blick auf die vielfältigen Anforderungen im Kontext von Zivilstatistik. Sie stellen auch für die universitäre Ausbildung von Lehramtsstudierenden anspruchsvolle Ziele dar. Gewisse Teile dieser Facetten lassen sich im Rahmen einer didaktischen Reduktion schon im Schulunterricht ansprechen. In ProCivicStat wurden 27 Lehreinheiten in deutscher Sprache (und noch weitere Einheiten in Englisch, Portugiesisch und Ungarisch) für den Unterricht in Schulen und Hochschulen erstellt und größtenteils auch 
in Seminaren getestet. Alle Arbeitsmaterialien haben ein konkretes gesellschaftlich relevantes Thema oder eine Frage als Ausgangspunkt und können im Unterricht z. T. ab Klassenstufe 9 oder 10 bis hin zum Universitätskurs innerhalb von 3 bis 8 Unterrichtsstunden bearbeitet werden. Die breite Palette an Themen umfasst Fragen wie z.B.: Wie ist das Verdiensteinkommen von Angestellten und Arbeitern in Ländern Europas und weltweit verteilt? Bekommen weibliche Angestellte weniger Gehalt als ihre männlichen Kollegen? Sind Menschen in manchen Ländern und Weltregionen glücklicher als anderswo? Hat die Kriminalitätsrate in Deutschland mit der Zuwanderung von Flüchtlingen zugenommen? Wird die Menschheit immer dicker? Bekommen dunkelhäutige Spieler im europäischen Fußball öfter rote Karten als ihre hellhäutigen Mitspieler? Unter welchen Bedingungen ist die Feinstaubbelastung in Stuttgart besonders hoch?

Nach einer inhaltlichen, kontextbezogenen Einführung und Verweisen auf weiterführende Informationen zur thematischen Vertiefung werden in diesen Materialien umfangreiche Datensätze mit Aufgaben zur Datenanalyse vorgestellt, die mit didaktisch konzipierten und frei verfügbaren digitalen Werkzeugen wie Fathom, CODAP, Gapminder, iNZight, im Netz verfügbaren Apps oder professioneller Software wie $\mathrm{R}$ oder Tableau bearbeitet werden können. Eine deutsche Version von Fathom kann kostenlos heruntergeladen werden (www.stochastik-interaktiv.de/fathom). CODAP (Common Online Data Analysis Platform, frei verfügbar unter codap.concord.org) ist eine didaktisch konzipierte Umgebung zum Datenmanagement und zur Visualisierung von Daten, ähnlich konzipiert wie Fathom, aber mit beschränkterem Leistungsumfang. CODAP ist web-basiert, ist daher nutzbar ohne Installierung von lokaler Software und ermöglicht die Bereitstellung von elektronischen Arbeitsblättern im Netz. iNZight (www.stat.auckland.ac.nz/ wild/iNZight/) ist eine R-basierte benutzerfreundliche Oberfläche zur statistischen Analyse und Datenvisualisierung, die von Chris Wild und Kollegen an der Universität in Auckland erstellt wurde. Frischemeier et al. (2016) geben einen kurzen Einblick in das Potential verschiedener Softwares beim Explorieren von zivilstatistischen Daten.

\subsection{Das Portal CivicStatMap}

Um die Auswahl geeigneter Unterrichtsmaterialien zu erleichtern, wurde im Projekt ProCivicStat die spezielle Plattform CivicStatMap (verfügbar über http://iaseweb.org/islp/pcs, siehe Abb. 4) entwickelt. Nach Auswahl eines interessierenden Themenbereichs (z. B. Migration, Climate and Environment, Income Inequalities, Education etc.), eines statistischen Inhalts (z.B. Mean, Boxplots, P-Value, Correlation etc.) und eines bevorzugten digitalen Hilfsmittels zur Analyse und Visualisierung (z. B. CODAP, Fathom, Gapminder, iNZight, Tableau, R) wird man direkt auf konkrete Lehrmaterialien inklusive Datensätze zum Einsatz im Unterricht verwiesen. Die Bearbeitung dieser Materialien vernetzt in der Regel neben Kontextwissen, grundlegendem mathematischen und statistischen Wissen auch statistische Kompetenzen, die im traditionellen Unterricht kaum behandelt werden, wie z.B. multivariates Denken, die Suche nach verborgenen Drittvariablen und Interaktionen sowie Simpsons Paradox, den Vergleich von Verteilungen, die Erkundung funktionaler Beziehungen zwischen Variablen und die Nutzung von unterschiedlichen 
Abb. 4 Ausschnitt aus CivicStatMap (https://rstudio.up.pt/ shiny/users/pcs/civicstatmap/)

\section{CivicStatMap}

CivicStatMap is a way of linking ideas, data sources, statistical concepts and visualization tools. Filter your selection and find the appropriate teachers and students material!

Note: You can select multiple statistical topics. To make multiple selection of statistical topics use the shift key.

Note: Below you will find the links to the interface for the 4 languages (Portuguese, English, German and Hungarian).

Portuguese Version

English Version

German version

Hungarian Version

Language:

German r.

Statistical_Topics:

AI(use shifit key for multiple selection)

Mean

Proportion

P-value

\section{Tools:}

CODAP, iNZight or iNZight Lite

Theme:

Happiness
Level_of_difficulty:
High

Material_typo:

All

$\checkmark$

Darstellungen und Visualisierungen. Die Bearbeitung fordert und fördert kritisches Denken: Wie wurden Variablen definiert? Wie wurden Konstrukte (z. B. Armutsrisiko oder Arbeitslosigkeit) operationalisiert? Auf welche Weise, aus welchem Grund und von wem wurden die Daten erhoben?

Exemplarisch stellen wir im Folgenden ein an der PH Ludwigsburg entwickeltes und getestetes Lehr-Lernmaterial vor, mit dem wir darstellen, wie Aspekte von $\mathrm{Zi}$ vilstatistik in der Lehre an Schule und Hochschule thematisiert werden können. Eine detaillierte Behandlung dieses Beispiels mit Hinweisen für Unterrichtende sowie ein Arbeitsblatt mit detaillierten Informationen kann mittels CivicStatMap mit folgenden Filtereinstellungen heruntergeladen werden (siehe Abb. 4): Language: German, Theme: Happiness, Level_of_difficulty: Intermediate; Material Type: Teachers. 


\subsection{Beispiel: Der World Happiness Report - Wie zufrieden sind wir?}

Zufriedenheit und Glück ist neben Reichtum und Gesundheit ein wichtiger Bestandteil für die meisten Menschen, wenn es um die Wünsche für das eigene Leben geht. Es wird auch zunehmend als das angemessene Maß für sozialen Fortschritt angesehen. Im Juni 2016 verpflichtete sich die OECD, bei Wachstumsberichten das Wohl der Menschen in den Mittelpunkt der Bemühungen der Regierungen zu stellen. In einer 2017 gehaltenen Rede sprach sich die Leiterin des UN-Entwicklungsprogramms (UNDP) gegen die so genannte „Tyrannei des BIP“ aus und argumentierte, dass es auf die Qualität des Wachstums ankomme. Der Weltglücksbericht (World Happiness Report) wurde in Zusammenarbeit mit den Vereinten Nationen erstmals 2012 veröffentlicht und misst jährlich wie glücklich die Menschen in den jeweiligen Ländern sind. Doch was bedeutet ,glücklich und zufrieden“ und wie kann man das messen? Und in welcher Beziehung steht Lebensglück zu anderen Variablen wie Gesundheit, Reichtum, soziale Eingebundenheit? Die Daten des World Happiness Reports sind frei verfügbar (http://worldhappiness.report/ed/2018/). Auf dieser Internetseite findet sich auch eine genaue Beschreibung, wie jede der 19 Variablen operationalisiert und gemessen wurde (siehe auch Tab. 1). Zum Beispiel wurde die Variable Glück als Selbsteinschätzung von befragten Personen auf einer Skala von 0 bis 10 gemessen. Im englischen Originaltext lautet die Frage wie folgt:

Please imagine a ladder, with steps numbered from 0 at the bottom to 10 at the top. The top of the ladder represents the best possible life for you and the bottom of the ladder represents the worst possible life for you. On which step of the ladder would you say you personally feel you stand at this time?

Tab. 1 listet einige zentrale Variablen auf und erläutert ihre Operationalisierung.

Die Operationalisierungen werfen eine Reihe von Fragen auf: Wie valide sind Selbsteinschätzungen? Einige Fragen werden kulturbedingt gewiss recht unterschiedlich interpretiert. Vom statistischen Standpunkt aus betrachtet ist die Definition von Großzügigkeit bemerkenswert: Ausgehend von der Annahme eines linearen

Tab. 1 Kurze Definition von einigen Variablen des World Happiness Report

\begin{tabular}{ll}
\hline Variable & Definition und Operationalisierung \\
\hline Glück & Selbsteinschätzung auf Skala von 0 bis 10 (siehe oben) \\
Wohlstand & Logarithmus des Bruttoinlandsprodukts pro Einwohner \\
Sozialer & „Wenn Du in Schwierigkeiten bist, hast Du jemanden, auf dessen Hilfe Du Dich verlas- \\
Rückhalt & sen kannst?“ (binäre Antwort 0 oder 1) \\
Gesundheit & $\begin{array}{l}\text { Basierend auf der Lebenserwartung errechnet aus Daten der WHO wird mittels einem } \\
\text { Faktor, der dem Verhältnis gesunde Lebensjahre zu gesamten Lebensjahren entspricht } \\
\text { (aus dem Jahr 2012), die zu erwartenden gesunden Lebensjahre bei der Geburt ermittelt }\end{array}$ \\
& $\begin{array}{l}\text { „Bist Du zufrieden oder unzufrieden mit Deiner Freiheit zu wählen, was Du mit Deinem } \\
\text { Freiheit }\end{array}$ \\
Geben machst?“ (Binäre Antwort 0 oder 1) \\
Großzügigkeit wird angegeben durch die Residuen der Regressionsgerade des nationalen \\
Dahr & $\begin{array}{l}\text { Despendet?“ in Relation zum Bruttoinlandsprodukt pro Kopf } \\
\text { Jahr der Datenerhebung }\end{array}$ \\
\hline
\end{tabular}




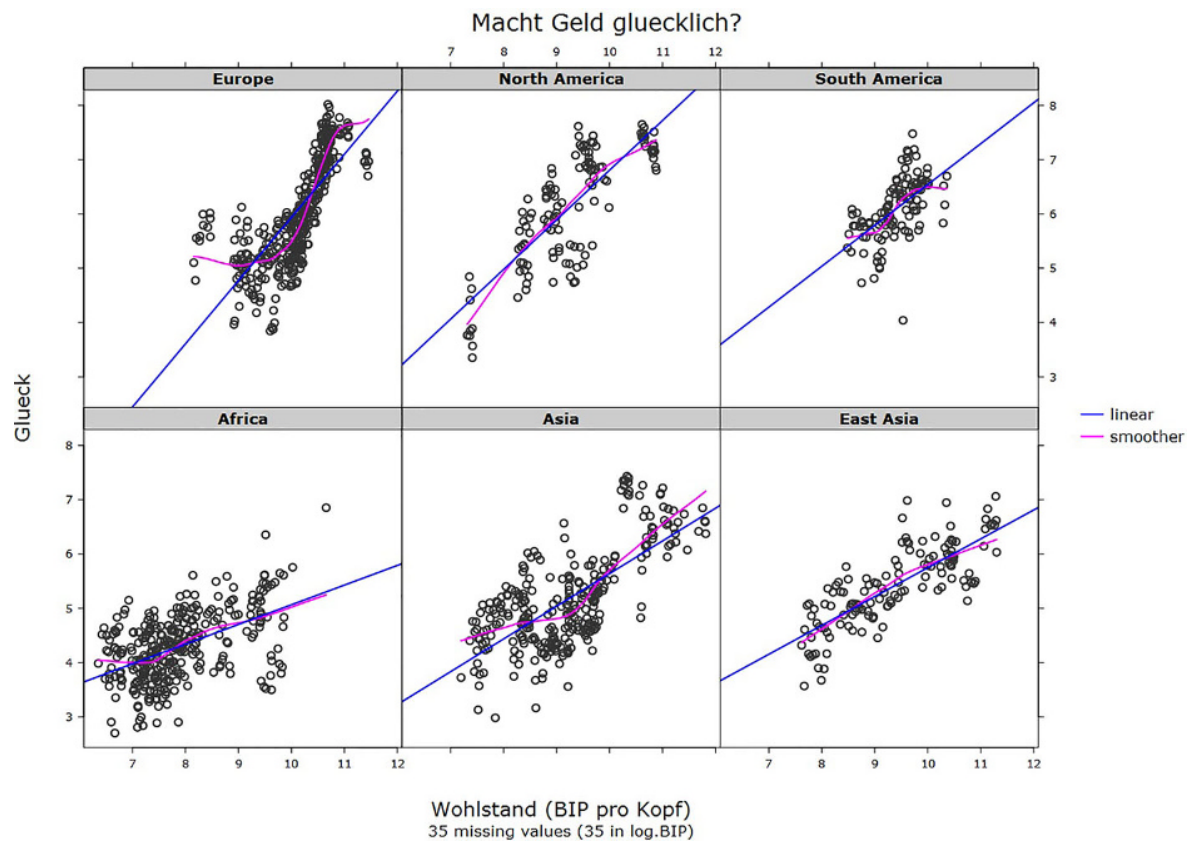

Abb. 5 Wohlstand versus Glück, jeweils getrennt für sechs Kontinente bzw. Weltregionen; jeder Punkt entspricht den (arithmetischen) Durchschnittwerten eines Landes bezogen auf ein Jahr zwischen 2005 und 2016; eingezeichnet sind die jeweiligen Regressionsgeraden sowie ein Datenglätter (loess mit Spannweite $\mathrm{h}=0,7)$

Zusammenhangs zwischen Wohlstand und Spendenbereitschaft wird Großzügigkeit als das Residuum der Regression von Spendenaufkommen relativ zum BSP pro Kopf definiert.

Macht Geld glücklich? Wir betrachten in Abb. 5 einige Visualisierungen zu dieser Frage (erstellt mit der Software iNZight).

Ein Zusammenhang zwischen Wohlstand und Glück auf dem Aggregationsniveau der Länderebene ist unverkennbar. Die Steigungen der Regressionsgeraden (als Indikator des Einflusses von Wohlstand auf Glück) schwankt deutlich von Kontinent zu Kontinent: In Afrika ist der Einfluss am geringsten (Steigung $\mathrm{m}=0,36$, Rangkorrelation $r=0,47$ ), in Europa hingegen am stärksten (Steigung $m=1,164$; Rangkorrelation $\mathrm{r}=0,87$, wie die Berechnungen mit iNZight anzeigen.

Aber in Europa gibt durchaus deutliche Abweichungen vom linearen Trend sowie Ausreißer. Abb. 6 zeigt ein Diagramm von Wohlstand versus Glück beschränkt auf die Länder Europas im Jahre 2016. Man beachte den sigmoiden Verlauf des Datenglätters (loess). Die sigmoide Form könnte man so deuten, dass sich Wohlstand erst aber einem bestimmten Level auf das Glücksempfinden auswirkt und bei einem bestimmten Wohlstand eine ,Sättigung“ eintritt. Menschen in skandinavischen Ländern wie Finnland scheinen besonders vom Glück, aber auch vom Wohlstand gesegnet, wohingegen das Glücksempfinden in Luxemburg unterhalb der vom Wohlstand genährten Erwartungen bleibt. Das könnte daran liegen, dass das BPI vielfach 
Macht Geld gluecklich?

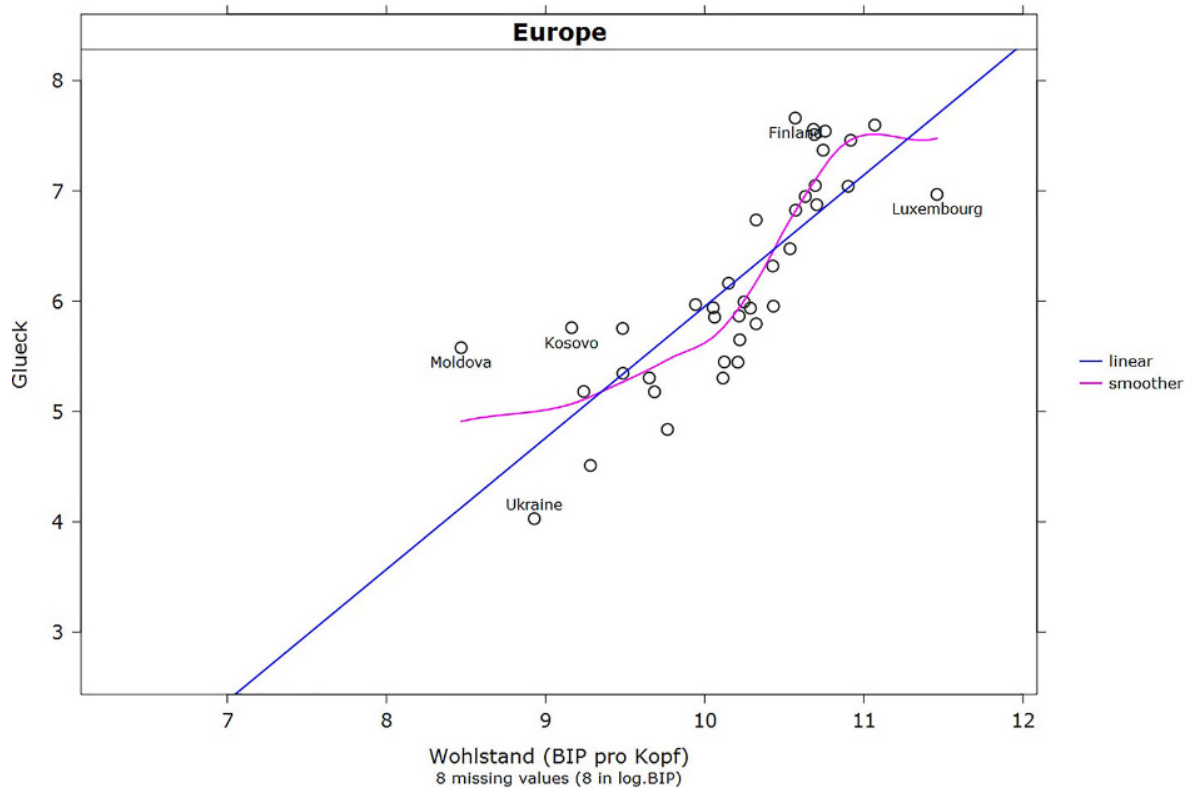

Abb. 6 Wohlstand versus Glück in den Ländern Europas im Jahre 2016. Jeder Punkt entspricht dem durchschnittlichen Glück (basierend auf Selbsteinschätzungen in der Stichprobe) und dem Bruttoinlandsprodukt pro Kopf

von ausländischen Konzernen erbracht wird, es sich nicht auf das Glücksempfinden der Bürger niederschlägt. Das Beispiel Ukraine lässt sich vermutlich dadurch erklären, dass die politischen Umstände und vor allem der Bürgerkrieg das erlebte Glück mindern. Die Graphik verweist auf zahlreiche Phänomene, die weiter zu klären wären.

Sind Menschen in reicheren Ländern großzügiger als Menschen in armen Ländern? Es ist klar, dass wer viel hat, sich auch größere Spenden leisten könnte. Aber nach obiger Operationalisierung des Begriffs Großzügigkeit wird dem größeren Wohlstand Rechnung getragen. Abb. 7 gibt Anlass zu der Vermutung, dass die Spendenbereitschaft mit steigendem Wohlstand stärker variiert (die Streuung der Daten wächst mit zunehmendem Wohlstand), und dass gerade bei Menschen in Ländern mit eher mittlerem Wohlstand die Großzügigkeit eher gering ist im Vergleich zu Ländern mit armer wie auch mit reicher Bevölkerung.

Während in Abb. 5 und 7 Daten über alle Jahre zwischen 2005 bis 2016 dargestellt werden, ist oft die zeitliche Entwicklung von Variablen von Interesse. Abb. 8 zeigt Boxplots pro Jahr der Variable Gesundheit (siehe Tab. 1), basierend auf Daten der Weltgesundheitsorganisation WHO. Wenn man berücksichtigt, dass die Stichprobe von $n=35$ Ländern im Jahre 2005 in den Folgejahren beständig anwuchs und seit 2011 mit kleinen Schwankungen bei $n=140$ Ländern liegt, und in den früheren Jahren vor allem in Ländern der nördlichen Hemisphäre ermittelt wurde, so lässt sich ein leichter Trend zur Erhöhung der Lebenserwartung über die Jahre hinweg 


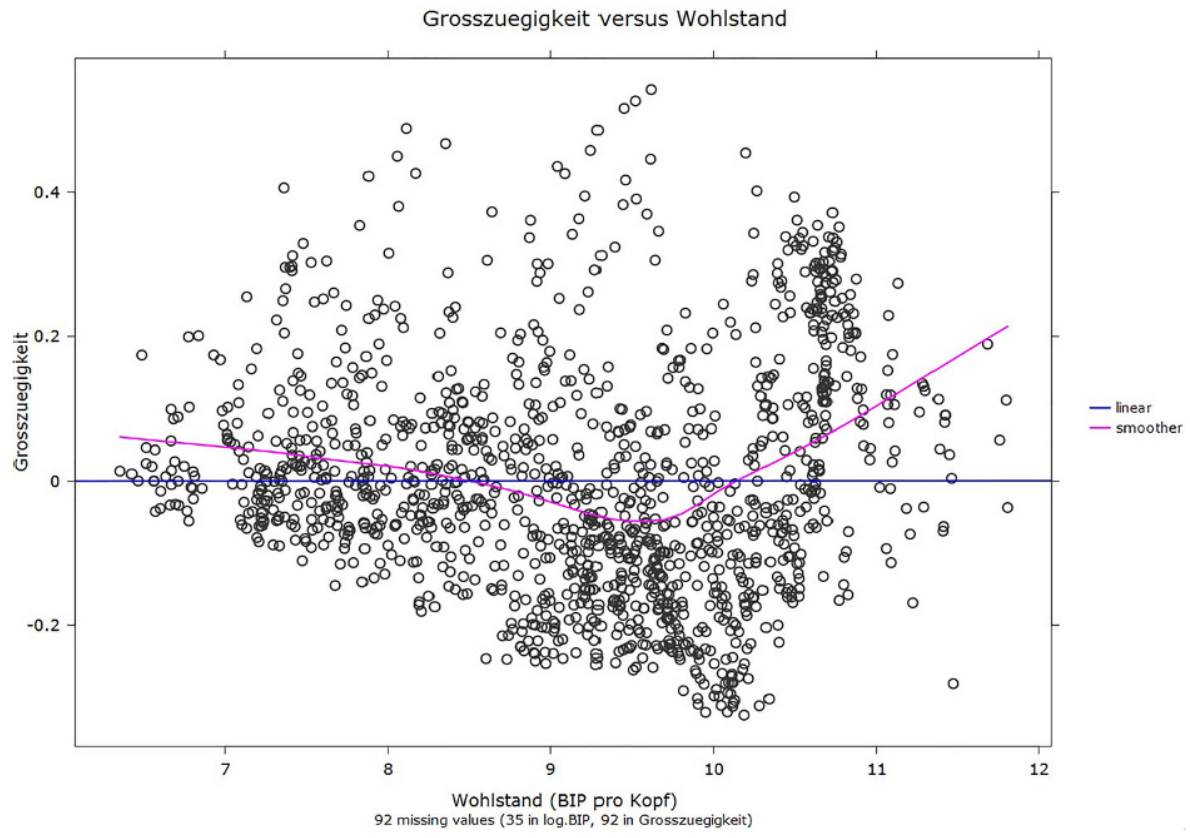

Abb. 7 Streudiagramm von Wohlstand versus Großzügigkeit; jeder Punkt entspricht den Durchschnittwerten eines Landes bezogen auf ein Jahr zwischen 2005 und 2016; eingezeichnet ist die Regressionsgerade sowie der Datenglätter (loess)

ablesen. Seit 2008 stieg der Median von 62,31 auf 64,79 im Jahre 2016 monoton an (numerische Zusammenfassung der Daten per iNZight).

Bei der Betrachtung der Entwicklung der Variable Gesundheit innerhalb der Jahre 2006 bis 2016 (2005 wird aufgrund deutlich geringerer Fallzahlen nicht weiter berücksichtigt) kann ein Trend zur Verbesserung erkannt werden (siehe Abb. 8). Das globale Minimum erhöht sich nahezu kontinuierlich von 36,17 (2006, Simbabwe) auf 43,38 (2016, Lesotho). Das globale Maximum erhöht sich von 74,28 (2006, Hongkong) auf 76,41 (2016, Singapur). Auch der Median und das arithmetische Mittel (letzteres ist nicht in Abb. 8 dargestellt, sondern mit Hilfe von iNZight als numerische Zusammenfassung errechnet) steigen nahezu kontinuierlich an, eine Ausnahme ist dabei das Jahr 2011.

Diese exemplarischen Erläuterungen verweisen auf das didaktische Potential des World Happiness Reports im Rahmen von Zivilstatistik. Das über das Portal CivicStatMap verfügbare Arbeitsblatt „Der World Happiness Report - Wie zufrieden sind wir" bietet weitere Detailinformationen und Aufgabenstellungen. Das schon in Abschn. 3 vorgestellte Netzdiagramm erlaubt für konkrete Aufgabenstellungen eine Bewertung, wie stark einzelne Facetten von Zivilstatistik sichtbar und Kompetenzen für die Bearbeitung erforderlich sind (von $0=$ überhaupt nicht bis $8=\mathrm{sehr}$ stark). Abb. 9 zeigt eine subjektiv vorgenommene Einschätzung, wie stark einzelne Facetten bei der Bearbeitung des Arbeitsblatts zum World Happiness Report vertreten sind. Ausgangspunkt ist die Frage, was Menschen glücklich macht und wel- 
Gesunde Lebenserwartung

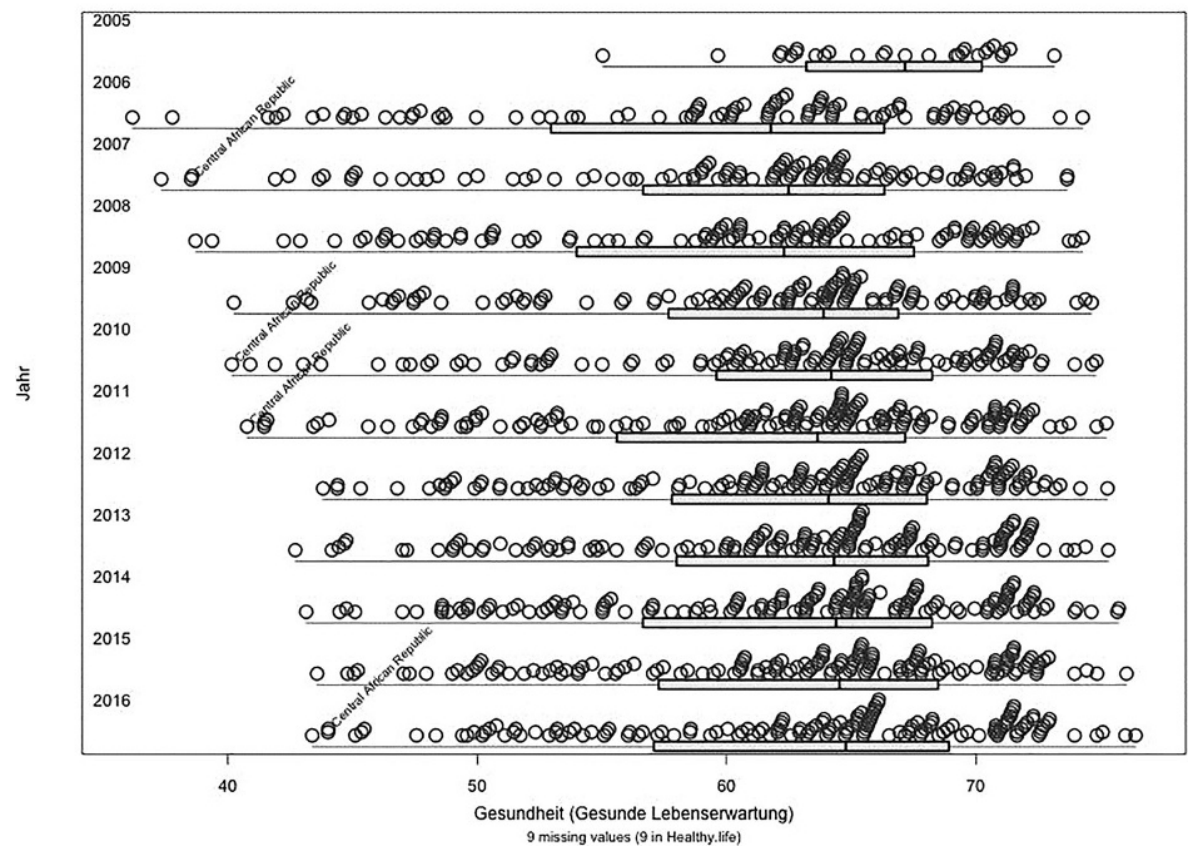

Abb. 8 Die Entwicklung der Variable „Gesundheit“ im Laufe der Jahre 2005-2016

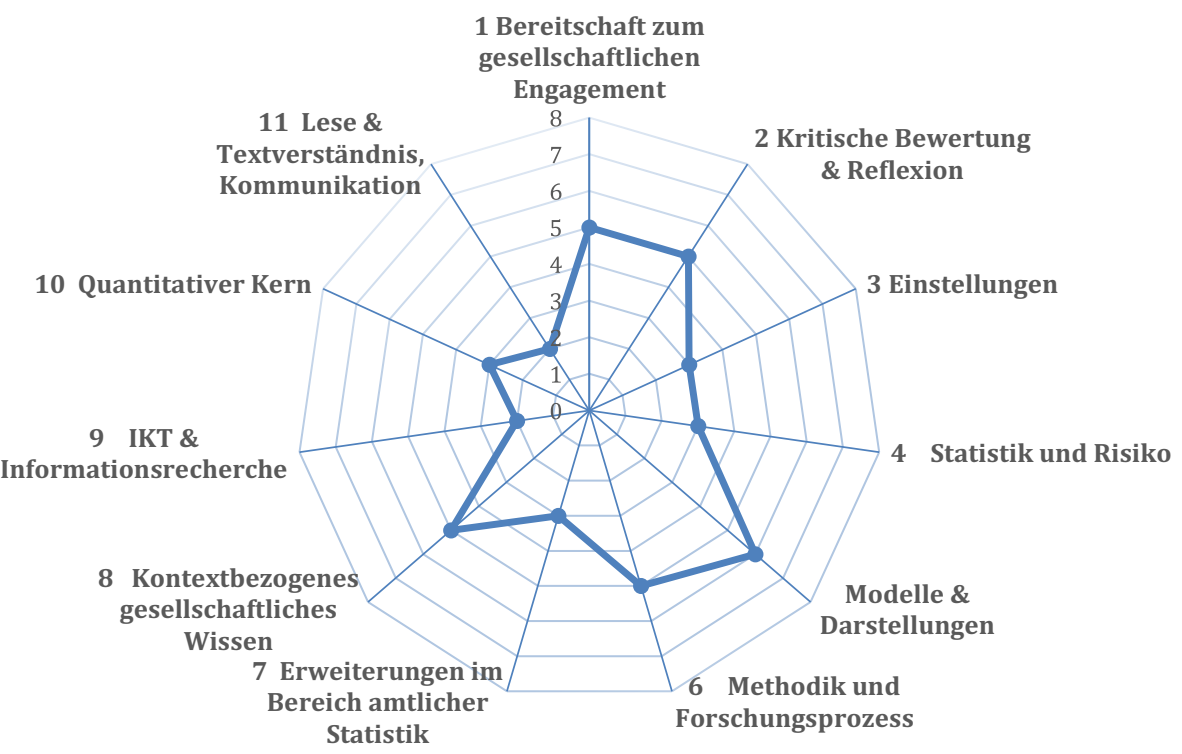

Abb. 9 Netzdiagramm zur Bewertung, wie stark einzelne Facetten in die Problemlösung hineinwirken 
che gesellschaftlichen Lebensumstände zum Glück der Menschen beitragen können (Facette 1). Die Untersuchungen hierzu benötigen Kenntnisse zur Darstellung und Modellierung (Facette 5) von schwer zu messenden Variablen. Hier sind kritische Bewertungen der Operationalisierung der Variablen (Facette 2) sowie eine realistische Einschätzung der Qualität der Erhebungsmethoden (Facette 6) gefordert. Kontextwissen zu verschiedensten Kulturen und ökonomischen Lebensbedingungen in verschiedenen Ländern (Facette 6) ist zur Bewertung und Interpretation der Darstellungen erforderlich während nach unserer (subjektiven) Einschätzung die anderen Facetten hier weniger gefordert sind.

\section{Curriculare Implikationen und bildungspolitische Empfehlungen}

Unsere Analysen im Projekt ProCivicStat weisen auf Mängel und Probleme der aktuellen Ausbildung in Statistik an Schulen und Hochschulen hin. Damit sich junge Menschen kompetent und informiert auf öffentliche Debatten zu gesellschaftlichen Themen einlassen und für eine Lösung brennender Probleme engagieren können, halten wir curriculare Änderungen in der Art, wie Statistik an Schulen und Universitäten gelehrt wird, für notwendig. Datenanalyse, Interpretation von Graphiken und (dynamische) Visualisierungen beziehen sich nicht alleine auf mathematische Techniken, sondern sind Teil des Erkenntnisgewinns in vielen Fächern und sollten daher Bestandteil eines breiten Fächerkanons sein. Lehre von Statistik darf sich nicht nur auf die Vermittlung von Techniken und formalen Begriffen mit wenig inhaltlichem Bezug beschränken, sondern kann bei zivilstatistischen Themen die Relevanz mathematisch begründeter Analysen illustrieren und junge Menschen somit befähigen, sich evidenzbasiert eine eigene fundierte Meinung zu bilden und sich in öffentliche Entscheidungsprozesse informiert einzubringen.

Wie lassen sich diese Konzepte in Curricula und Bildungsgängen an Schulen und Hochschulen umsetzen? Im Abschlussbericht des Projektes ProCivicStat (ProCivicStat Partners 2018) werden unter dem Titel „Engaging Civic Statistics: A Call for Action and Recommendations" sechs bildungspolitische und curriculare Empfehlungen formuliert, die sich auf das Lehren und Lernen von Statistik an allgemeinbildenden Schulen, Hochschulen und Universitäten beziehen. Wir schließen den Artikel mit diesen bildungspolitischen und curricularen Empfehlungen und geben hier eine kurze Zusammenfassung in deutscher Sprache. Der vollständige 40-seitige Report ist über die Webseite http://iase-web.org/islp/pcs verfügbar.

In der folgenden Empfehlung 1 geht es um den Kern von Zivilstatistik: Der Fokus liegt auf sozialen Phänomenen und sozialen Fragen. Die Empfehlungen 2 bis 5 untersuchen Teilbereiche, die sich auf didaktische Umsetzungsprobleme beziehen: Den Einsatz von Ressourcen für Lehrkräfte (d.h. Datensätze, Textdokumente und Pressemitteilungen), Lehrpläne, digitale Technologien, didaktische Konzepte, Bewertungen von Schülerleistungen usw. In Empfehlung 6 werden Faktoren und relevante Akteure im Rahmen nötiger systemischer Veränderungen angesprochen. Die aufgeführten Empfehlungen stellen einen vorläufigen Zwischenstand aus dem Projekt ProCivicStat dar. Wir laden daher zu weiteren Reaktionen ein und fordern alle an Statistikausbildung Interessierten zur weiteren Diskussion auf. 
Empfehlung 1: Lehr-Lernaktivitäten in Statistik sollen insbesondere auch die Auseinandersetzung mit sozialen, gesellschaftlich relevanten Themen fördern und das kritische Verständnis der Lernenden für statistische Analysen zu wichtigen gesellschaftlichen Phänomenen fördern.

Lehrpläne in Statistik sollten daraufhin untersucht werden, inwieweit sie Lernende befähigen und ermutigen, sich auf gesellschaftsrelevante Themen einzulassen.

Aktivitäten sollten

- eine klare Begründung für Inhalte und Lernaktivitäten haben, basierend auf einem konzeptionellen Rahmen, der ein breites Spektrum statistischer Fähigkeiten widerspiegelt, insbesondere in Bezug auf soziale und gesellschaftlich relevante Fragen,

- das Verständnis für die Rolle von statistischer Evidenz bei der Entscheidungsfindung in der Öffentlichkeit fördern - und insbesondere Konzepte der Unsicherheit, des Risikos und des Nutzens sowie der Wechselbeziehungen zwischen ihnen thematisieren.

Empfehlung 2: Es sollen relevante Daten und Texte verwendet und die multivariate, dynamische und aggregierte Natur sozialer Phänomene soll thematisiert werden.

Das Verständnis sozialer Phänomene und das Nachdenken über die Auswirkungen möglicher politischer Entscheidungen für das gesellschaftliche Sozialgefüge erfordern die Fähigkeit, mit multivariaten Daten zu argumentieren. In vielen traditionellen Lehreinheiten werden ausschließlich künstliche Daten verwendet. Lernende müssen über die wichtigsten Datenquellen, die Qualität und Vergleichbarkeit der Daten Bescheid wissen.

Aktivitäten mit Daten sollten

- Inhalte zu wichtigen gesellschaftlichen Themen für die Menschheit aufnehmen (z. B. Klimawandel, Ungleichheit und Migration),

- auf Daten von wichtigen Datenanbietern (z. B. Eurostat, UN und OECD) zugreifen und diese nutzen,

- mehrere Datenquellen verwenden, insbesondere auch neue Datenquellen (einschließlich Big Data und Open Data und Daten aus sozialen Medien), um Probleme triangulieren zu können - und dabei Fragen der Messbarkeit des in der Wirtschaftsstatistik bekannten Adäquationsproblems (z. B. Menges 1964) berücksichtigen.

Empfehlung 3: Es sollen Software und digitale Technologien eingesetzt werden, die vielseitige Visualisierungen und Interaktionen mit Daten zu relevanten sozialen Phänomenen ermöglichen.

Aktivitäten sollten relevante aktuelle und innovative Software und digitale Technologien in ihre Tätigkeiten als Werkzeug im Analyseprozess einbeziehen. Dies schließt ein

- die Verwendung dynamischer und interaktiver Datenvisualisierungen,

- Lernende zu befähigen, unbekannte Werkzeuge anzuwenden und zu bewerten einschließlich Werkzeuge aus Data Science, 
- von Lernenden die Verwendung verschiedener Instrumente für den Zugriff und die Analyse von Daten sowie die Darstellung ihrer Analysen und Schlussfolgerungen zu verlangen.

Empfehlung 4: Lehrmethoden sollen Fähigkeiten zur kritischen Interpretation einer Vielzahl von Daten- und Textquellen entwickeln.

Aktivitäten in der Zivilstatistik unterscheiden sich stark von traditionellen Kursen in Statistik oder Seminaren zu Forschungsmethoden und Sozialstatistik und zielen auf die Entwicklung von Fähigkeiten zum statistischen Argumentieren ab.

Aktivitäten sollten

- verschiedene Unterrichtsstrategien anwenden, wobei der Schwerpunkt auf aktiven Lernansätzen liegt, bei denen die Lernenden Fragen formulieren, begründete Nachweise für Behauptungen finden und geeignete Analysemethoden auswählen,

- Fähigkeiten zur kritischen Interpretation durch Analyse einer Vielzahl von Quellen (einschließlich Zeitungsartikeln und „Fake News“) entwickeln,

- Lernende ermutigen, sich über soziale Themen zu verständigen, indem sie narrative Berichte über komplexe Situationen erstellen, die auf mehreren statistischen Datenquellen basieren,

- schon früh im Kurs Argumentationen mit nichtlinearen und mit multivariaten Daten einführen, die für wichtige soziale Fragen relevant sind,

- Modellierungsfähigkeiten entwickeln, damit Lernende auf die Stärken und Schwächen von Instrumenten zur Modellierung sozialer Situationen aufmerksam werden,

- Lernende auf vielfältige Weise ansprechen, wie Nachweise zur Unterstützung von Theorien in verschiedenen akademischen Disziplinen verwendet werden.

Empfehlung 5: Assessments sollen die Fähigkeit überprüfen, Daten, statistische Befunde und Nachrichten zu wichtigen sozialen Phänomenen zu untersuchen und kritisch zu verstehen.

Die Beurteilungen und Rückmeldungen, die Lernende während und am Ende eines Kurses erhalten, sind für sie der stärkste Leitfaden zur Orientierung darüber, was zu lernen ist und inwieweit sie Fortschritte erzielen.

Aktivitäten sollten

- (formative und summative) Beurteilungen enthalten, die die zentralen Bildungsziele von Zivilstatistik widerspiegeln. Insbesondere sollte die Fähigkeit evaluiert werden, Datenanalysen mit ihrer gesellschaftlichen und politischen Bedeutung in Beziehung zu setzen,

- mit den Bewertungssystemen vereinbar sein, die es den Lernenden ermöglichen, die unter Empfehlung 4 empfohlenen Fähigkeiten unter Verwendung der unter Empfehlung 3 empfohlenen Inhalte und Werkzeuge unter Beweis zu stellen, z. B. über Projektarbeit oder Portfolios, Videos, Präsentationen oder andere nicht-traditionelle Medien, sowohl als Einzelpersonen wie auch in Gruppenarbeit.

Empfehlung 6: Die Förderung des zivilstatistischen Verständnisses erfordert systemische Veränderungen und die Zusammenarbeit relevanter Akteure. 
Zivilstatistik braucht institutionelle Unterstützung, um zur praktischen Realiät zu werden. Dies erfordert einen kohärenten Plan für systemische Änderungen. In einigen Kontexten könnte dies damit beginnen, dass überwiegend traditionelle Kurse Elemente von Zivilstatistik aufnehmen. In anderen Kontexten kann es sinnvoll sein, authentische, große Datensätze für soziale Fragestellungen zu verwenden, um traditionelle statistische Inhalte zu vermitteln. In anderen Zusammenhängen kann es erforderlich sein, radikale Lehrplanreformen durchzuführen.

Bildungseinrichtungen sollten

- einzelne Kurse und Studienprogramme überprüfen und beurteilen, inwieweit zivilstatistische Themen angesprochen werden,

- sicherstellen, dass zivilstatistische Themen ein wesentliches Element in der Lehreraus- und weiterbildung sind und dass Lehrerinnen und Lehrer die Herausforderung erfahren, aus komplexen Datensätzen Schlussfolgerungen zu ziehen,

- Lehrende und Fakultäten ermutigen, sich für Forschungsprojekte im Zusammenhang mit Zivilstatistik zu engagieren und ihnen Gelegenheit dazu zu geben,

- die Zusammenarbeit zwischen Lehrenden in verwandten Disziplinen fördern,

- die Zusammenarbeit mit offiziellen Statistikanbietern und den Medien fördern.

\section{Schlussbemerkungen}

Der Aufsatz fasst die Ergebnisse des ProCivicStat Projekts zusammen, auch um die Leserinnen und Leser zu motivieren, sich die ausführlichen Unterrichtsmaterialien und theoretischen Analysen anzusehen. Die im Projekt kooperierenden Partner bereiten eine Buchveröffentlichung vor, in denen die einzelnen Komponenten des Projektes weiter ausgearbeitet werden. Dieser Artikel stellt eine gründliche Erweiterung und adressatenorientierte Umarbeitung der Artikel zum Thema in Stochastik in der Schule, 2019, 39(1) dar, die sich an Mathematiklehrkräfte richten. Einige Passagen sind wörtlich übernommen, ohne sie als solche explizit zu kennzeichnen.

Förderung Die Vorbereitung dieses Beitrags wurde in Teilen finanziert mit Unterstützung der Europäischen Kommission. Diese Veröffentlichung stellt lediglich die Ansichten der Verfasser dar und die Kommission ist nicht verantwortlich für irgendwelche Ansichten, die sich aus den hier geäußerten Informationen ergeben.

\section{$\stackrel{*_{* * * *}^{* *}}{* * *^{*}}$ Erasmus+}

Open Access Dieser Artikel wird unter der Creative Commons Namensnennung 4.0 International Lizenz veröffentlicht, welche die Nutzung, Vervielfältigung, Bearbeitung, Verbreitung und Wiedergabe in jeglichem Medium und Format erlaubt, sofern Sie den/die ursprünglichen Autor(en) und die Quelle ordnungsgemäß nennen, einen Link zur Creative Commons Lizenz beifügen und angeben, ob Änderungen vorgenommen wurden. Die in diesem Artikel enthaltenen Bilder und sonstiges Drittmaterial unterliegen ebenfalls der genannten Creative Commons Lizenz, sofern sich aus der Abbildungslegende nichts anderes ergibt. Sofern das betreffende Material nicht unter der genannten Creative Commons Lizenz steht und die betreffende Handlung nicht nach gesetzlichen Vorschriften erlaubt ist, ist für die oben aufgeführten Weiterverwendungen des Materials die Einwilligung des jeweiligen Rechteinhabers einzuholen. Weitere Details zur Lizenz entnehmen Sie bitte der Lizenzinformation auf http://creativecommons.org/licenses/by/ 4.0/deed.de. 


\section{Literatur}

Ben-Zvi D, Garfield J (2004) The challenge of developing statistical literacy, reasoning and thinking. Springer Netherlands, Dordrecht

Borovcnik M, Ossimitz G (1987) Materialien zur Beschreibenden Statistik und Explorativen Datenanalyse. Hölder-Pichler-Tempsky, Wien

De Condorcet A (1994) Foundations of social choice and political theory. Edward Elgar, Brookfield (Original publiziert 1792)

Engel J (Hrsg) (2016) Promoting understanding of statistics about society. In: Proceedings of the Roundtable Conference of the International Association of Statistics Education (IASE), Berlin, July 2016

Engel J (2019) Statistical Literacy und Gesellschaft: Was ist Zivilstatistik? Stoch Sch 1(39):2-12

Fischer R (1984) Offene Mathematik und Visualisierung. Math Didact 7:139-160

Fischer R (2001) Höhere Allgemeinbildung. In: Fischer A, Fischer-Buck A, Schäfer KH, Zöllner D (Hrsg) Situation-Ursprung der Bildung. Franz-Fischer-Jahrbuch der Philosophie und Pädagogik, Bd. 6. Universitätsverlag, Leipzig, S 151-161

Freire P (1973) Pädagogik der Unterdrückten. Bildung als Praxis der Freiheit. Rowohlt, Reinbek

Frischemeier D, Biehler R, Engel J (2016) Competencies and dispositions for exploring micro data with digital tools. In: Engel J (Hrsg) Promoting understanding of statistics about society Berlin, Germany, July 2016. Proceedings of the Roundtable Conference of the International Association of Statistics Education (IASE)

GAISE (2016) Guidelines for assessment and instruction in statistics education: college report. American Statistical Association. https://www.amstat.org/asa/education/Guidelines-for-Assessment-andInstruction-in-Statistics-Education-Reports.aspx. Zugegriffen: 30. Juni 2019

Gal I (2002) Adults' statistical literacy: meanings, components, responsibilities. Int Stat Rev 70(1):1-51

Heidrich J, Bauer P, Krupka D (2018) Strukturen und Kollaborationsformen zur Vermittlung von Data-Literacy-Kompetenzen - Stand der Forschung. Arbeitspapier Nr. 32. https:// hochschulforumdigitalisierung.de/sites/default/files/dateien/HFD_AP_Nr32_Data_Literacy_ Kompetenzen_Literatur.pdf. Zugegriffen: 30. Juni 2019

Krüger K (2012a) Haushaltsnettoeinkommen - ein Beispiel zur Nutzung der GENESIS-Online Datenbank im Unterricht. Stoch Sch 32(3):8-14

Krüger K (2012b) Was die Arbeitslosenzahlen(nicht)zeigen - Interpretation von Daten der Bundesagentur für Arbeit. Mathematikunterricht 58(4):32-41

Lesser L (2007) Critical values and transforming data: teaching statistics with social justice. J Stat Educ 15(1):1-21

Loos A (2018) Data science in the news business. In: Biehler R, Budde L, Frischemeier D, Heinemann B, Podworny S, Schulte C, Wassong T (Hrsg) Paderborn Symposium on Data Science Education at School Level 2017: The collected extended abstracts. Universitätsbibliothek Paderborn, Paderborn, S $44-45$

Menges G (1964) Ätilität und Adäquation. Stat Hefte 22(2):144-149

Nicholson J, Gal I, Ridgway J (2018) Understanding civic statistics: a conceptual framework and its educational applications. A product of the procivicstat project. http://IASE-web.org/islp/pcs. Zugegriffen: 30. Juni 2019

OECD (2009) Focus on citizens: public engagement for better policy and services. http://www10.iadb.org/ intal/intalcdi/pe/2009/03785.pdf. Zugegriffen: 30. Juni 2019

ProCivicStat Partners (2018) Engaging civic statistics: a call for action and recommendations. A product of the procivicstat project. http://IASE-web.org/ISLP/PCS. Zugegriffen: 30. Juni 2019

Ridgway J (2015) Implications of the data revolution for statistics education. Int Stat Rev 84(3):528-549

Ridgway J, Nicholson J, Stern D (2017) Statistics education in a post-truth era. In: Molnar A (Hrsg) Teaching statistics in a data rich world Proceedings of the Satellite conference of the International Association for Statistical Education (IASE), Rabat, Morocco, July 2017

Ridsdale C, Rothwell J, Smit M, Ali-Hassan H, Bliemel M, Irvine D, Wuetherick B (2015) Strategies and best practices for data literacy education: knowledge synthesis report. http://hdl.handle.net/10222/ 64578. Zugegriffen: 30. Juni 2019

Rinsdorf L, Boers R (2016) The need to reflect: data journalism as an aspect of disrupted practice in digital journalism and journalism education. In: Engel J (Hrsg) Promoting understanding of statistics about society Proceedings of the Roundtable Conference of the International Association of Statistics Education (IASE), Berlin, Germany, July 2016

Schield M (2011) Statistical literacy: a new mission for data producers. Stat J IAOS 27:173-183 
Skovsmose O (1994) Towards a philosophy of critical mathematics education. Springer, Heidelberg, Berlin, New York

de Smedt M (2016) European Statistics and Eurostat's contribution to improving statistical literacy. In: Engel J (Hrsg) Promoting understanding of statistics about society Proceedings of the Roundtable Conference of the International Association of Statistics Education (IASE), Berlin, July 2016

Vohns A (2016) Mathematical literacy as a civil right and/or civic duty? Two general education approaches. Paper presented at the International Conference on Mathematics Education (ICME 13)_TSG 23, Hamburg. https://www.researchgate.net/publication/294729397_Mathematical_Literacy_as_ a_Civil_Right_andor_a_Civic_Duty_Two_General_Education_Approaches. Zugegriffen: 30. Juni 2019

Vohns A (2017) Bildung, mathematical literacy and civic education: The (strange?) case of contemporary Austria and Germany. In: Chronaki A (Hrsg) Mathematics Education and Life at Times of Crisis. Proceedings of the Ninth International Mathematics Education and Society Conference, Bd. 2. University of Thessaly Press, Volos, S 968-978

Wallman K (1993) Enhancing statistical literacy: enriching our society. J Am Stat Assoc 88(421):1-8

Watson JM (2006) Statistical literacy at school: growth and goals. Erlbaum, Mahwah

Weiland T (2017) Problematizing statistical literacy: an intersection of critical and statistical literacies. Educ Stud Math 96:33-47

Hinweis des Verlags Der Verlag bleibt in Hinblick auf geografische Zuordnungen und Gebietsbezeichnungen in veröffentlichten Karten und Institutsadressen neutral. 